\title{
1 A combined EM and proteomic analysis places HIV-1 Vpu at the crossroads of retromer and ESCRT
}

2 complexes: PTPN23 is a Vpu-cofactor

3 Short title: Spatial proteomics identifies Vpu targets and cofactors

4 Charlotte A. Stoneham ${ }^{1 *}$, Simon Langer ${ }^{2}$, Paul D. De Jesus ${ }^{2}$, Jacob M. Wozniak ${ }^{3}$, John Lapek ${ }^{3}$, Thomas

5 Deerinck ${ }^{4}$, Andrea Thor ${ }^{4}$, Lars Pache², Sumit K. Chanda², David J. Gonzalez ${ }^{3}$, Mark Ellisman ${ }^{4,5}$, John

$6 \quad$ Guatelli ${ }^{1 *}$

$7 \quad{ }^{1}$ Department of Medicine, University of California, San Diego School of Medicine and Veterans Affairs

8 San Diego Healthcare System, La Jolla, California, USA

$9{ }^{2}$ Infectious and Inflammatory Disease Center, Sanford Burnham Prebys Medical Discovery Institute,

1010901 North Torrey Pines Road, La Jolla, California, USA

$11{ }^{3}$ Department of Pharmacology, Skaggs School of Pharmacy and Pharmaceutical Sciences, University of

12 California, San Diego, La Jolla, California, USA

$13{ }^{4}$ National Center for Microscopy and Imaging Research, Center for Research on Biological Systems,

14 University of California, San Diego, School of Medicine, La Jolla, California, USA

$15{ }^{5}$ Department of Neurosciences, University of California, San Diego School of Medicine, La Jolla,

16 California, USA

17 *correspondence: cstoneham@health.ucsd.edu, jguatelli@health.ucsd.edu 


\section{$\underline{\text { Abstract }}$}

The HIV-1 accessory protein Vpu modulates membrane protein trafficking and degradation to provide evasion of immune surveillance. Targets of Vpu include CD4, HLAs, and BST-2. Several cellular pathways co-opted by Vpu have been identified, but the picture of Vpu's itinerary and activities within membrane systems remains incomplete. Here, we used fusion proteins of Vpu and the enzyme ascorbate peroxidase (APEX2) to compare the ultrastructural locations and the proximal proteomes of wild type Vpu and Vpu-mutants. The proximity-omes of the proteins correlated with their ultrastructural locations and placed wild type Vpu near both retromer and ESCRT-0 complexes. Hierarchical clustering of protein abundances across the mutants was essential to interpreting the data and identified Vpu degradationtargets including CD4, HLA-C, and SEC12 as well as Vpu-cofactors including HGS, STAM, clathrin, and PTPN23, an ALIX-like protein. The Vpu-directed degradation of BST-2 required PTPN23 but not the retromer subunits. These data suggest that Vpu directs targets from sorting endosomes to degradation at multi-vesicular bodies via ESCRT-0 and PTPN23.

\section{Author Summary}

32 Vpu triggers the degradation or mis-localization of proteins important to the host's immune response.

33 Vpu acts as an adaptor, linking cellular protein targets to the ubiquitination and membrane trafficking machinery. Vpu has been localized to various cellular membrane systems. By fusing wild type Vpu and Vpu-mutants to the enzyme ascorbate peroxidase, we defined the cellular proteome in proximity to Vpu and correlated this with the protein's location. We found that wild type Vpu is proximal to ESCRT

37 proteins, retromer complexes, and sorting and late endosomal proteins. Functionally, we found that the 38 Vpu-mediated degradation of the innate defense protein BST-2 required PTPN23, an ALIX-like protein, consistent with our observation of Vpu's presence at the limiting membranes of multi-vesicular bodies. 


\section{Introduction}

41 HIV-1 encodes the accessory proteins Vif, Vpr, Nef, and Vpu to overcome cell-intrinsic, innate, and

42 adaptive host defenses. Vpu is a small, non-enzymatic, integral membrane protein that functions as an

43 adaptor, linking targeted cellular proteins to the protein quality control and membrane trafficking

44 machinery to induce their re-localization or degradation. Cellular targets of Vpu interfere directly with

45 viral replication or support immune surveillance; these targets include CD4 (the virus's primary

receptor), BST-2 (an interferon-induced protein that traps newly assembled virions on the infected-cell

47 surface), natural killer (NK) cell receptors (NTB-A), class I MHC (HLA-C), CCR7, and tetraspanins (1-7).

Several Vpu cofactors and co-opted pathways have been identified. These include a Skp1/cullin1/F-box

(SCF) multi-subunit E3 ubiquitin ligase containing $\beta$ - $\operatorname{TrCP}(8)$. A phospho-serine acidic cluster (PSAC) in

the cytoplasmic domain (CD) of Vpu binds $\beta$-TrCP, recruiting the E3 ligase and inducing polyretains newly synthesized BST-2 in the trans-Golgi network (TGN), and it inhibits the recycling of endocytosed BST-2 to the plasma membrane $(12,13)$. The net down-regulation of BST-2 from the cell

57 surface requires clathrin and partly depends on the hetero-tetrameric clathrin adaptor (AP) complexes 1

58 and $2(10,14-16)$. An acidic leucine-based motif and the PSAC in Vpu's CD bind the AP complexes

59 directly, enabling Vpu to redirect the endosomal transport of its targets independently of the SCF E3

60 ubiquitin ligase $(10,15-18)$. Nonetheless, the endo-lysosomal degradation of BST-2 depends on Vpu's

61 PSAC as well as the SCF E3 ligase, and on the ubiquitin-binding protein HRS (HGS), a subunit of the 
63 The multifaceted mechanism of Vpu-action is reflected in the protein's complex subcellular itinerary,

64 which includes the ER, plasma membrane (PM), and various endosomal compartments. Consistent with

65 this, microscopic data place Vpu at steady-state in the ER, Golgi and the TGN, the PM, and recycling

66 endosomes $(9,20-22)$.

67 We asked whether a more integrated view of Vpu-activity could be obtained via a high-depth

68 characterization of the protein's proximal proteome. Proximity-labeling covalently tags protein-

69 neighbors in living cells with small molecules such as biotin, enabling isolation by affinity-purification

70 and identification and quantitation by mass spectrometry. This approach enables the identification of

71 physiologically relevant interactions even when they are weak or transient. In the present study, we

72 expressed Vpu fused to the enzyme APEX2, an ascorbate peroxidase whose catalytic activities enable

73 both electron microscopic localization as well as the labeling of proximal proteins with biotin (23). The

74 biotinylated proteins were captured, identified, and quantified using tandem-mass-tag (TMT)-based

75 proteomics. To correlate ultrastructural localization with proximal-proteins, we compared wild type Vpu

76 to three Vpu-mutants: Vpu-A18H, a mutant of the Vpu transmembrane domain (TMD) that is retained in

77 the ER; Vpu-AAA/F, a mutant of the alanine face of the Vpu TMD that interacts with the TMD of BST-2

78 and other Vpu targets; and Vpu-S52,56N, a mutant of the key serines of the PSAC motif, which is unable

79 to interact with either $\beta-\operatorname{TrCP}$ or the medium $(\mu)$ subunits of AP-1 and AP-2 and is partly displaced from

80 juxtanuclear endosomes to the plasma membrane.

81 Through these comparisons, we characterized the proximal proteome and itinerary of Vpu, including

82 changes in response to the above substitutions in Vpu whose consequences for protein-interaction and

83 function are partly known. Our results place wild type Vpu at sorting endosomes, from which its targets

84 can be diverted via ESCRT-related proteins toward the interior of multivesicular bodies for degradation. 
85 The results generate hypotheses regarding potential Vpu targets, such as SEC12, and they identify

86 PTPN23 as a novel cofactor of Vpu.

\section{$87 \quad \underline{\text { Results }}$}

\section{Vpu-APEX2 fusion is well expressed and functional.} intracellular protein imaging by electron microscopy and spatially resolved protein mapping (Martell,

The enzyme ascorbate peroxidase 2 (APEX2) was genetically fused to the C-terminus of FLAG-tagged human codon-optimized clade B (NL4.3) Vpu (VpHu) with a short intervening linker sequence (Fig. 1A). APEX2 is modified for enhanced activity and improved detection sensitivity compared to its predecessor, APEX (23). Given the size of the tag (28 kDa) compared to that of Vpu (17 kDa), we first tested whether the fusion to APEX2 impaired Vpu activity. When transiently expressed in HeLa P4.R5 cells, which express the HIV receptors CD4, CXCR4, and CCR5 as well as BST-2, the Vpu(FLAG)-APEX2 fusions were well-expressed as measured by western blotting (Fig. 1B). Vpu-induced downregulation of surface BST-2 and CD4 was measured by immunofluorescent staining and flow cytometry (Fig.1). APEX2-tagged Vpu retained biological activity against BST-2 and CD4, although it was slightly less active than Vpu tagged only with a C-terminal FLAG-epitope (Fig. 1C).

\section{Vpu-APEX2 distorts juxtanuclear endosomes and labels the limiting membranes of multi-vesicular} bodies (MVBs).

APEX enables the generation of electron-dense material in fixed cells that can be detected by transmission electron microscopy (24). An advantage of APEX over horseradish peroxidase (HRP) is that it maintains activity in the reducing cytosolic environment. This allows APEX to be used for both Deerinck et al. 2012). 
The subcellular distribution of wild-type Vpu-APEX2 was first evaluated by electron microscopy (Fig. 2).

107 HeLa cells were transiently-transfected to express Vpu-FLAG or Vpu-FLAG-APEX2. The next day, the cells

112 Most of these vesicles were unilamellar, enlarged vesicles (EVs), but some were consistent with multi-

113 vesicular bodies (MVBs). The Vpu-APEX2 stain was restricted to the limiting membrane of MVBs and did

114 not appear on intra-lumenal vesicles. Vesiculated Golgi was observed in cells transfected with Vpu-FLAG

115 (without the APEX2 tag; Fig. 2C). In contrast, in non-transfected HeLa P4.R5 cells, the Golgi appeared as

116 a typical stack of flattened cisternae (Fig. 2D). These data are consistent with Vpu's known activities and

117 residence in biosynthetic endo-lysosomal membranes $(14,15,25,26)$.

Localization of Vpu-APEX2 mutants: Vpu-A18H mis-localizes to the ER; Vpu-S52,56N mis-localizes to

121 when both constructs contained previously characterized mutations. We evaluated three Vpu-mutants:

122 Vpu-A18H, a mutant of the Vpu transmembrane domain (TMD) that is retained in the ER (26); Vpu-

$123 \mathrm{AAA} / \mathrm{F}$, a mutant of the alanine face of the Vpu TMD that interacts with the TMD of BST-2 and

124 potentially other Vpu targets (27); and Vpu-S52,56N, a mutant of the key serines of the PSAC motif (8).

125 HeLa P4.R5 cells transfected to express Vpu WT or the Vpu mutants tagged with FLAG were fixed and

126 stained for the FLAG epitope and the trans Golgi resident marker protein TGN-46, then visualized by

127 immunofluorescence microscopy (Fig. 3A). Vpu-FLAG localized to juxtanuclear membranes and

128 overlapped partially with TGN-46, in agreement with previous studies $(15,20,22)$. As anticipated, Vpu 
A18H was restricted to the nuclear envelope and a cytoplasmic, ER-like distribution. The AAA/F mutation did not alter the localization of Vpu appreciably. In contrast, Vpu-S52,56N displayed a relatively more dispersed cytoplasmic staining.

Similar distributions were observed at the light microscopic level when Vpu-APEX2 and the mutants were visualized using $\mathrm{H}_{2} \mathrm{O}_{2}, \mathrm{DAB}$, and osmium (Fig. 3B). The distribution of WT Vpu-APEX2 was juxtaand peri-nuclear, whereas Vpu-A18H-APEX2 was ER-like. In contrast, Vpu-S52,56-APEX2 outlined the cell perimeter, consistent with residence at the plasma membrane. Electron microscopic images (Fig. 3C) revealed vesiculation of juxtanuclear membranes by the $A A A / F$ and $S 52,56 \mathrm{~N}$ mutants as well as by the wild type Vpu-APEX2 (Fig. S1). Vpu-A18H-APEX2 stained the nuclear envelope and ER membranes and at high levels of expression induced a striking alteration in the structure of these organelles (Fig. S2). VpuS52,56N-APEX2 stained the plasma membrane, consistent with the light-microscopic observations.

These data indicated that the subcellular distribution of Vpu-APEX2 was similar to that of Vpu without the APEX-tag. The data also indicated that specific mutations in Vpu-APEX2 modulated the protein's subcellular distribution in a manner consistent with known properties of Vpu.

\section{The Vpu-proximity-ome defined by multiplexed quantitative proteomics and comparison of Vpu} mutants.

In living cells and in the presence of hydrogen-peroxide, APEX can catalyze the generation of biotinphenoxy radicals to enable rapid, spatially restricted labeling of proximal proteins; these proteins can then be isolated by standard pull-down methods and identified by mass spectrometry. An advantage of APEX-based proximity labeling is that weak interactions can be identified, which would otherwise be lost during standard affinity purification. Moreover, neighboring proteins should be labeled and identified, even if they are not direct interactors. To evaluate the feasibility of this approach in the case of Vpu, we first detected proteins biotinylated by Vpu(FLAG)-APEX2 using immunofluorescence and immunoblot. 
HeLa P4.R5 cells were transiently-transfected to express Vpu(FLAG)-APEX2 or the previouslycharacterized Mito-Matrix-APEX2 (23). The next day, the cells were incubated with hydrogen peroxide in the presence of biotin-phenol for 1 minute, fixed, and stained with streptavidin conjugated to the fluorescent label Alexa Fluor 594 (Fig. 4B). In cells transfected to express Vpu-APEX2, streptavidin was concentrated in perinuclear regions and overlapped with FLAG, consistent with biotinylation of proteins in close proximity to Vpu-APEX2, likely including Vpu-APEX2 itself. The streptavidin signal was also present faintly and diffusely throughout the nucleus and cytoplasm. In contrast, in cells transfected to express Mito-APEX2, streptavidin was restricted to mitochondrial structures without a diffuse background. This restriction presumably reflects the generation of biotin-phenoxy radicals within the enclosed membranes of mitochondria in the case of Mito-APEX2 rather than in the cytosol in the case of Vpu-APEX2. In a second set of experiments, cells expressing Vpu(FLAG)-APEX2 and related mutants, or expressing Mito-APEX2, were incubated with hydrogen peroxide in the presence of biotin-phenol for 1 minute, lysed, and the proteins were separated by SDS-PAGE and analyzed by western blot. Biotinylated proteins were detected using streptavidin conjugated to HRP. The size distribution of proteins biotinylated by Vpu-APEX2 was strikingly different from that of Mito-APEX2, but the distribution of proteins biotinylated by WT Vpu-APEX2 and the related Vpu-mutants were indistinguishable. For the

168 Vpu proteins, the most abundant band at $40 \mathrm{kDa}$ likely corresponds to self-biotinylation of Vpu-APEX2.

169 Minimal background biotin labeling was observed in cells transfected with empty plasmid (control) or $170 \mathrm{Vpu}(\mathrm{FLAG})$ lacking the APEX2 tag.

171 For our preliminary mass spectrometry experiments, we transfected HeLa P4.R5 cells to express the WT 172 Vpu-APEX2, or the mutants, A18H, AAA/F, S52,56N, or the Mito-APEX2 control. The next day, the APEX2-

173 catalyzed biotinylation procedures were performed, and biotinylated proteins were captured on

174 streptavidin-coated beads. The captured proteins were eluted from the beads and analyzed by 175 quantitative proteomics. Data were normalized as described in the Materials and Methods and are 
presented as a heatmap of the relative abundance of 1779 common proteins identified in all samples

177 (Fig. S3A). As anticipated, the Mito-APEX2 induced biotinylation of mitochondrial proteins, reflected in the high relative enrichment of proteins which conform to the Gene Ontology (GO) term "mitochondrial matrix" and other mitochondrion-associated cellular components (Fig. S3B, S3C).

In two subsequent independent experiments, the Vpu WT and mutants were compared for differential enrichment of biotinylated proteins that might represent potential cofactors or targets (Fig. 5 and Fig. statistical parameters were used to compare the relative enrichment of proteins between Vpu WT and the mutants, as the fold changes were relatively low; data are presented as the relative protein enrichment in the presence of Vpu mutants and wild-type $\mathrm{Vpu}$, with $p$-value determined by $t$-test. The proximity-ome of the $\mathrm{A} 18 \mathrm{H}$ mutant was enriched for proteins derived from the biosynthetic pathway

187 (such as SEC proteins) and COPII-coated vesicles, while depleted in plasma membrane and endosomal 188 proteins relative to that of wild type $\mathrm{Vpu}$. These data were consistent with the ultrastructural imaging, 189 which demonstrated restriction of the $\mathrm{A} 18 \mathrm{H}$ mutant to predominantly the ER and nuclear envelope. The AAA/F mutant, whose ultrastructural distribution was similar to that of the WT Vpu, had few proteins

191 significantly enriched or depleted relative to the WT protein (data not shown). In contrast, the 192 proximity-ome of the $\mathrm{S} 52,56 \mathrm{~N}$ mutant, which at the light and EM level was partially redistributed to the 193 plasma membrane, was significantly enriched in plasma membrane proteins including EGFR and known 194 targets of Vpu, (CD4, CD81, and HLA-C), while depleted in endosomal proteins relative to that of wild 195 type Vpu.

196 K-means clustering analysis of the patterns of protein enrichment across the four conditions (WT Vpu 197 and the three mutants) highlighted potential cofactors (cluster 6) and targets (cluster 5) for Vpu 198 activities (Fig. 6.) Specifically, we reasoned that cluster 5 proteins, which were decreased relative to WT 
when Vpu was retained in the ER by the $\mathrm{A} 18 \mathrm{H}$ mutation but increased when Vpu was displaced to the plasma membrane by the $\$ 52,56 \mathrm{~N}$ mutation, would include Vpu targets, especially if degraded by an ERAD-like mechanism. Consistent with this notion, cluster 5 includes known Vpu targets CD4, CD81, A18H mutation and the $552,56 \mathrm{~N}$ mutation, could reflect the local intracellular environment of WT Vpu. complex (Vps35 and SNX3), among others. These data place wild type Vpu at the sorting endosome, a crossroads in the endosomal system at which Vpu is well-positioned to inhibit recycling and target proteins to endo-lysosomal degradation.

\section{Potential novel Vpu targets.}

210 We reasoned that proteins enriched in proximity to Vpu-S52,56N relative to WT Vpu could also

211 represent proteins that are stabilized when Vpu is mutated and might be targets of Vpu-mediated

212 degradation. To distinguish such targets of Vpu-mediated degradation from proteins that instead reflect

213 changes in the local environment of Vpu induced by the displacement of the $552,56 \mathrm{~N}$ mutant to the

214 plasma membrane, we first measured surface-downregulation using flow cytometry. HeLa P4.R5 cells

215 were transfected to express Vpu-FLAG, and surface EGFR, CD55, and CD4 were measured (data not

216 shown). CD4 was downregulated by Vpu as expected, but EGFR and CD55 were unaffected; these

217 proteins likely become more proximal to $\mathrm{Vpu}$ when $\mathrm{Vpu}$ is displaced to the plasma membrane and are

218 unlikely to be a degradation-targets.

219 To screen further for Vpu targets, we used the relatively high throughput Global Arrayed Protein

220 Stability Analysis (GAPSA) assay (28). cDNAs encoding approximately 160 proteins that were significantly

221 enriched in proximity to the S52,56N mutant relative to WT Vpu were screened for degradation by WT 
222 Vpu, using Vpu-S52,56N as a control. We found eight proteins that were significantly degraded by WT

223 Vpu but not by the S52,56N mutant (Fig. 7). Degradation of these proteins was confirmed by western

224 blotting of the V5-tagged target proteins. Three of these proteins, CD4, HLA-C, and CD99, are known

225 Vpu targets, but the others, including PREB/SEC12, are newly identified.

\section{Potential novel Vpu cofactors.}

227 We reasoned that proteins enriched in proximity to WT Vpu relative to Vpu-S52,56N might represent

228 cellular cofactors, as the $\mathrm{DS}_{52} \mathrm{GxxS}_{56}$ sequence has been shown to be a relatively promiscuous motif with

229 regard to recruiting both the SCF-E3 ligase and clathrin AP complexes $(8,16,18)$. STRING analysis of

230 proteins identified in k-means cluster 6, which includes proteins whose proximity to Vpu is decreased by

231 the S52,56N substitution, revealed a network of interrelated proteins involved in ubiquitin sorting,

232 endosomal trafficking, and retromer-mediated trafficking (Fig. 8A).

233 We investigated whether Vpu-FLAG colocalized with these proteins by immunofluorescence microscopy.

234 HeLa P4.R5 cells were transfected to express Vpu-FLAG then fixed and stained for FLAG and either

235 STAM, PTPN23, or Vps35; each colocalized with Vpu (Fig. 8B). We also observed partial colocalization

236 between Vpu and the retromer protein SNX3 (Fig. S4).

237 To examine whether these proteins have roles in Vpu activities, we tested the ability of Vpu to degrade

238 BST-2 in their absence. HeLa P4.R5 cells were depleted of STAM, Vps35, SNX3, or PTPN23 using siRNAs

239 before transfection with proviral plasmids encoding either wild type HIV-1 clone NL4-3 (encoding Vpu)

240 or NL4-3 $\triangle \mathrm{Vpu}$. Total cellular BST-2 was measured by western blotting 24 hours after transfection of the

241 proviral plasmids (Fig. 8C). As expected, NL4-3 encoding Vpu reduced the levels of BST-2 compared to

242 NL4-3AVpu in cells treated with the control siRNA (siNeg) (Fig. 8C). Knockdown of the ESCRT-0 subunit

243 STAM markedly increased the steady-state levels of BST-2, but Vpu still reduced those levels.

244 Knockdown of PTPN23, an ALIX-like protein involved in the formation of intralumenal vesicles in MVBs 
245 (29), inhibited the Vpu-mediated degradation of BST-2 without substantially affecting BST-2 levels in the

246 absence of Vpu. Depletion of the retromer proteins Vps35 and SNX3 had minimal if any effect on Vpu-

247 mediated degradation of BST-2. These data support distinct roles for ESCRT-0 and PTPN23 in the

248 degradation of BST-2: whereas the ESCRT-0 protein STAM supported physiologic degradation of BST-2 in

249 the absence of Vpu, PTP23N instead supported Vpu-mediated degradation specifically, consistent with

250 activity as a Vpu cofactor.

\section{Discussion}

252 Proteomic methods have been used to explore host-HIV protein-protein interactions and to look broadly

253 at changes in the plasma membrane induced by the virus $(30,31)$. Here, we used proximity labeling

254 mediated by APEX2 fusion proteins as a novel approach to define the proteome proximal to the HIV-1

255 protein $\mathrm{Vpu}$, a membrane protein that mediates viral evasion of innate and adaptive immunity. The use

256 of Vpu mutants whose subcellular localization differed was key to distinguishing proteins specific to the

257 neighborhood of wild type Vpu from a large background. We observed that Vpu-APEX2 (like Vpu without

258 the APEX2 fusion-tag) distorted juxtanuclear endosomes, replacing typical stacks of thin Golgi cisternae

259 with enlarged vesicles. These vesicles, as well as the limiting membranes of MVBs, were labeled when

260 cells expressing Vpu-APEX2 were visualized by thin section electron microscopy. When Vpu was

261 mutationally trapped in the ER, its proximal proteome included an abundance of ER-associated proteins

262 including components of COPII coats. When Vpu was mutationally displaced to the plasma membrane,

263 its proximal proteome was depleted of early and sorting endosomal components; these included

264 subunits of the retromer and the ESCRT-0 complexes, as well as the ALIX-like protein PTPN23, which

265 supported the degradation of BST-2 by Vpu. Comparison of the proximal proteomes of the wild type and

266 mutant Vpu proteins yielded a list of proteins up-regulated by substitution of the serines within the

267 protein's PSAC motif. Some of these are likely proximity markers of the plasma membrane, consistent 
with the Vpu mutant's change in localization, while others, including HLA-C, CD99, and SEC12, are potentially subject to Vpu-directed degradation, a possibility supported by the transient expression experiments herein.

271 Many proteins identified here are unlikely to be either Vpu targets or cofactors, but nonetheless

272 contribute to creating a picture of Vpu's itinerary within cellular membranes. That itinerary seems

273 focused on sorting endosomes identified by the specific presence of EEA1 and ESCRT-0 subunits in the 274 neighborhood of wild type Vpu. However, it also likely includes late endosomes and MVBs, consistent 275 with the presence of the ALIX-like protein PTPN23, which supports the budding of intralumenal vesicles 276 into MVBs (29). This characterization of Vpu is reinforced by the labeling of the limiting membranes of 277 MVBs by wild type Vpu-APEX2 when the enzyme is used to generate an osmiophilic reaction product.

278 PTPN23 is required for the sorting of EGFR into MVBs and its ultimate degradation (32). Consistent with 279 this, functional data herein indicate that PTPN23 is a cofactor of Vpu on the path of endo-lysosomal degradation of at least one of its targets, BST-2. Whether PTPN23 directly interacts with Vpu remains an 281 open question. Nonetheless, PTPN23 does not seem required for the physiologic degradation of BST-2, 282 suggesting that it defines a distinct pathway of degradation co-opted by Vpu. Although not found in the 283 heatmap of Figure 6 (see Supplemental Table 2), the PTPN23-associated protein CHMP 4B and the 284 deubiquitinase USP8/UBPY co-clustered with PTPN23 and STAM in the independent experiments shown 285 in supplemental figure S2 (see Supplemental Table 1)(32). These data are consistent with a model in 286 which Vpu co-opts ESCRT-0, PTPN23, and CHMP 4B to direct targets into the lumen of MVBs for 287 degradation. PTPN23, knockdown of STAM markedly increased the expression of BST-2 in either the absence or presence of Vpu. This suggests that ESCRT-0, which plays a key role in the sorting of ubiquitinated 
291

292

293

294

295

296

297

298

299

300

301

302

303

304

305

306

307

308

309

310

311

membrane proteins from early endosomes to late endosomes at the expense of recycling to the plasma membrane (33), constitutively targets BST-2 toward endo-lysosomal degradation. Vpu presumably acts downstream of that step, since it can stimulate partial degradation of BST-2 even when the expression of BST-2 is increased by knockdown of STAM.

Although our primary intention was not to look for new Vpu-targets, our data suggest several novel targets of serine-dependent Vpu-mediated degradation. One of the more intriguing is PREB, also known as SEC12, a guanine nucleotide exchange factor for the GTPase Sar1p (34), which regulates the formation of COPII coats and ER-to-Golgi transport. Degradation of SEC12 by Vpu, shown here in transient expression experiments, could cause a block in ER-to-Golgi transport and is potentially consistent with the reported inhibition of exocytic membrane trafficking by $\vee p u(12,35)$. Although it might also be consistent with the formation of the large juxtanuclear endosomes observed electron microscopically, those structures were not serine-dependent (Fig. S1). On the other hand, cells expressing the ER-restricted Vpu-A18H mutant often showed exuberant accumulation of ER membranes emanating from the nuclear envelope, potentially consistent with an exaggerated SEC12 degradation phenotype and a block in ER-to-Golgi transport (Fig. S2).

In summary, correlative microscopic and proteomic analyses have provided a view of the Vpu-proximal proteins with unprecedented depth. The data place wild type Vpu predominantly at early sorting endosomes as well as at late endosomes and MVBs. The data generate new models, including the role of PTPN23 in degradation directed by Vpu at the MVB and the possibility that Vpu-mediated degradation of SEC12 underlies inhibition of exocytic trafficking. Elaborating these new models will require viral expression of Vpu in natural host cells such as CD4-positive T cells or macrophages. 


\section{Materials and Methods}

313 Cells: HeLa P4.R5 cells, which express the HIV-1 receptors CD4 and CCR5, were obtained from the NIH

314 AIDS Research and Reference Reagent program from Dr. Nathaniel Landau (36). HEK293 cells were

315 obtained from Dr. Saswati Chaterjee (City of Hope). HEK293T cells (used in GAPSA assays) were

316 purchased from ATCC (Manassas, VA). All cell lines were maintained in Dulbecco's modified Eagle

317 medium (DMEM) supplemented with 10\% fetal bovine serum (FBS), penicillin/streptomycin, and 1

$318 \mu \mathrm{g} / \mathrm{mL}$ puromycin in the case of HeLa P4.R5 cells.

319 Plasmids: The C-terminally FLAG-tagged human codon-optimized clade B (NL4.3) Vpu (VpHu) has been

320 previously described (17). The pCG-GFP reporter plasmid (37) was provided by Dr. Jacek Skowronski,

321 Case Western Reserve University, Cleveland, OH. pcDNA3.1-Connexin43-GFP-APEX2 construct was

322 obtained from Addgene, deposited by Dr. Alice Ting, Massachusetts Institute of Technology, Cambridge,

323 MA (23). The pcDNA3.1-based Vpu-FLAG-APEX2 was generated by overlap extension PCR amplification

324 before restriction digest and ligation into the pcDNA3.1(-) plasmid backbone between Nhel and EcoRI

325 sites. Vpu-FLAG-containing fragment was amplified using 5' AGATTCGCTAGCATGGTGCCCATTATTGTCGC

326 and 5' CACAGTTGGGTAAGACTTTCCGGAGCCGCCGCCCTTATCGTCGTCATCCTTGTAA primers, and FLAG-

327 APEX2 was amplified using 5'

328 TTACAAGGATGACGACGATAAGGGCGGCGGCTCCGGAAAGTCTTACCCAACTGTG and 5’

329 TGCTTAGAATTCTTAGGCATCAGCAAACCCAAG. Vpu-FLAG plasmid constructs encoding the mutations

$330 \mathrm{AAA} / \mathrm{F}, \mathrm{A} 18 \mathrm{H}$ and $\mathrm{S} 52,56 \mathrm{~N}$ were previously generated. The overlap extension PCR method was used to

331 amplify and ligate these mutated DNAs into the pcDNA3.1(-) backbone with a C-terminal APEX2 tag. The

332 Mito matrix-v5-APEX2 construct was generated in the Ting lab (23). Expression plasmids containing V5-

333 tagged cDNAs in the pLX304 backbone were obtained from the Lenti ORFeome Collection (38). A

334 pcDNA4-Vpu-FLAG plasmid was used for the GAPSA assay; LacZ-FLAG was used as a control (28). 
pcDNA4-Vpu-S52,56N-FLAG was generated by G-block synthesis (Integrated DNA Technologies, IDT) and ligation between BamHI and Notl sites of the pcDNA4 backbone, using In-fusion cloning reagent (Takara Bio). Full-length HIV-1 and HIV-1 lacking Vpu were expressed from the HIV-1 proviral plasmid pNL4-3 (39) and $p N L 4-3 \Delta \mathrm{Vpu}(40)$.

siRNAs: The siRNAs targeting STAM and Vps35 were custom synthesized by Sigma-Aldrich, the target

340 sequences were as follows; STAM: UAACUUGGUAUAUAAGGAAAGGGCC, and Vps35:

341 GCCUUCAGAGGAUGUUGUAUCUUUA. An siRNA targeting SNX3 was acquired from Dharmacon, target

342 sequence: CGUGACUAUUAAUGAUUGA. A validated siRNA targeting PTPN23 was purchased from

343 Thermo Fisher Scientific (s24775). The AllStars negative control siRNA was used as a non-specific control

344 (Qiagen).

\section{Transfections:}

346 Plasmids: Cells were transfected 24 hours after plating, using Lipofectamine 2000, following the

347 manufacturer's protocol (Invitrogen). Lipofectamine 2000 was diluted in Opti-MEM (Gibco) and

348 incubated for 5 minutes at RT prior to mixing with DNA diluted in Opti-MEM. The DNA:Lipofectamine

349 mix was incubated for 20 minutes prior to addition to cells in antibiotic-free media. The cells were

350 incubated with the transfection mix for 4 hours before the media was replaced.

351 siRNAs: Cells were reverse-transfected (transfected while plating) in 6-well plates $\left(3.5-5 \times 10^{5}\right.$ cells per

352 well) using Lipofectamine RNAimax transfection reagent (Invitrogen), following standard protocols. The

353 siRNAs were diluted in Opti-MEM, and added to wells containing cells in antibiotic-free media at a 10

$354 \mathrm{nM}$ final concentration. Assays were performed 48 or 72 hours post-transfection with siRNAs, as

355 indicated in figure legends. 
Flow Cytometry: To quantify cell-surface levels of CD4 and BST-2, HeLa cells transfected to express Vpu constructs or empty plasmid control, and the pCG-GFP transfection marker, were washed with $1 \mathrm{X}$ phosphate-buffered saline (PBS) and resuspended using Acutase dissociation media (Innovative Cell Technologies). The cells were collected and pelleted by centrifugation at $300 \times \mathrm{g}$ for 5 minutes, then resuspended in $100 \mu \mathrm{L}$ flow cytometry buffer (2\% FBS in PBS, and $0.1 \%$ sodium azide) and stained using either Alexa-647-conjugated mouse anti-BST-2 antibody, Alexa-647-IgG isotype control, APC-conjugated mouse anti-CD4, or APC-conjugated mouse IgG1 isotype control (BioLegend), and incubated for 30 minutes on ice. The cells were washed and pelleted three times before fixation in $2 \%$ paraformaldehyde 364 (PFA) in 1X PBS for 15 minutes. Surface BST-2 or CD4 was quantified using a BD Accuri C6 flow cytometer and CFlow Sampler analysis software. Data are presented as mean fluorescence intensity of FL4 (far-red) signal in the GFP-positive (FL1) cell population.

Immunofluorescence Microscopy: 1.2 × $10^{5} \mathrm{HeLa}$ P4.R5 cells were seeded on $12 \mathrm{~mm}$ coverslips in 24well plates 24 hours prior to transfection. Cells were transfected with 200 ng DNA using Lipofectamine 2000, following the manufacturer's protocol. The cells were fixed and stained the following day. The cells were washed in cold PBS and fixed in 4\% PFA in PBS on ice for 5 minutes, then 15 minutes at room

371 temperature (RT). The cells were washed twice with PBS and PFA was quenched with $50 \mathrm{mM}$

372 ammonium chloride for 5 minutes. The cells were permeabilized with $0.2 \%$ Triton X-100 in $1 X$ PBS for 7

373 minutes and blocked with $2 \%$ bovine serum albumin (BSA) for 30 minutes at RT prior to incubation with

374 primary antibodies for 2 hours at RT. Vpu was detected with mouse anti-FLAG (Sigma-Aldrich); the trans-

375 Golgi was detected with goat anti-TGN46 (ABD Serotec); Mito-V5-APEX2 was detected using mouse anti-

376 V5 (Invitrogen); biotin was detected with Alexa-594-conjugated Streptavidin (Invitrogen). Endogenous

377 protein cofactors were detected using rabbit anti-STAM antibody (ProteinTech), rabbit anti-PTPN23

378 (ProteinTech) goat anti-Vps35 (Novus Bio) and rabbit anti-SNX3 (Abcam). 
The cells were washed and stained with donkey anti-mouse rhodamine-X (RhX) or donkey anti-sheep AlexaFluor-488 (Jackson ImmunoResearch) for 1 hour at RT. For detection of APEX2 biotinylation by immunofluorescence, the cells were incubated with $500 \mu \mathrm{M}$ biotinyl-tyramide in pre-warmed medium for 30 minutes prior to addition of hydrogen peroxide $(1 \mathrm{mM})$. The cells were incubated for 1 minute prior to quenching with APEX2 quencher solution (see below), and washing $3 x$ with PBS before fixation and staining as above. Stretavidin conjugated to Alexa-Fluor 594 was used to detect biotinylated proteins. Following immunostaining, the cells were washed extensively in PBS, and briefly in water, before mounting in Mowiol (polyvinyl alcohol) mounting medium (prepared in-house). microscope fitted with a Hamamatsu CCD camera. For each field, a Z-series of images was collected, deconvolved using a nearest-neighbor algorithm (Slidebook software v6, Imaging Innovations, Inc) and presented as Z-stack projections. Image insets displaying colocalization are single Z-plane images. Image brightness was adjusted using Adobe Photoshop CS3.

Transmission Electron Microscopy: 6 × 105 HeLa P4.R5 cells were seeded in $35 \mathrm{~mm}$ poly-lysine-coated MatTek dishes and transfected 24 hours later with $1 \mu \mathrm{g}$ total pcDNA3.1-VpHu-FLAG-APEX2 or pcDNA3.1VpHu-S52,56N-FLAG-APEX2. 16 hours later, the cells were fixed in 2\% glutaraldehyde (Electron $\mathrm{mM}$ sodium cacodylate with $2 \mathrm{mM} \mathrm{CaCl}_{2}$ to quench unreacted fixative. The cells were washed with 100 $399 \mathrm{mM}$ sodium cacodylate with $2 \mathrm{mM} \mathrm{CaCl}_{2}$ and diaminobenzidine (DAB) staining was initiated with the 400 addition of freshly diluted $0.5 \mathrm{mg} / \mathrm{mL} \mathrm{DAB}$ (Sigma; from a stock of the free base dissolved in $0.1 \mathrm{M} \mathrm{HCl}$ ) 
stopped with the removal of the DAB solution, and the cells were again washed with $100 \mathrm{mM}$ sodium cacodylate with $2 \mathrm{mM} \mathrm{CaCl}$. Post-fixation staining was performed with $2 \%(\mathrm{w} / \mathrm{v})$ osmium tetroxide

404 (Electron Microscopy Sciences) for 30 minutes in chilled buffer. Cells were rinsed $5 \times 2$ minutes each in chilled distilled water and then placed in chilled $2 \%(\mathrm{w} / \mathrm{v})$ uranyl acetate in ddH2O (Electron Microscopy Sciences) overnight. Cells were washed in distilled water, and dehydrated in graded ethanol series (20\%, $50 \%, 75 \%, 90 \%, 95 \%, 100 \%, 100 \%, 100 \%)$, for 2 minutes each. The cells were brought to RT in $100 \%$ ethanol, and infiltrated with Durcapan ACM resin (Sigma-Aldrich) diluted in ethanol 1:1 for one hour.

409 The cells were then infiltrated with $100 \%$ resin twice for one hour each before curing at $60^{\circ} \mathrm{C}$ for 48 410 hours. DAB positive cells were identified at low resolution by wide-field microscopy. 70-90 nm-thin

411 sections were imaged using FEI-Tecnai G2 Spirit or JEOL 1200EX transmission electron microscopes

412 operating at $80 \mathrm{kV}$. Sample processing and imaging by electron microscopy was performed at the

413 National Center for Microscopy and Imaging Research at UC San Diego.

414 Western Blot: Cell monolayers were washed 3 times in ice-cold PBS and lysed in extraction buffer $(0.5 \%$

415 Triton X-100, $150 \mathrm{mM} \mathrm{NaCl}, 25 \mathrm{mM} \mathrm{KCl}, 25 \mathrm{mM}$ Tris, pH 7.4, 1 mM EDTA) supplemented with a protease

416 inhibitor mixture (Roche Applied Science). Extracts were clarified by centrifugation $(12,000 \times \mathrm{g}$ for 10

417 minutes at $4^{\circ} \mathrm{C}$ ). The sample protein concentration was determined by Bradford assay (BD Biosciences)

418 using standard protocols, and $10 \mu \mathrm{g}$ denatured by boiling for 5 minutes in SDS sample buffer. Proteins in

419 the extracts were resolved by SDS-PAGE using $12 \%$ or $4-15 \%$ gradient (BioRad) acrylamide gels,

420 transferred to PVDF membranes, and probed by immunoblotting using mouse anti-Actin (Sigma-

421 Aldrich), mouse anti-FLAG (Sigma-Aldrich), mouse anti-V5 (Invitrogen), STAM (ProteinTech), Vps35

422 (Novus Bio), SNX3 (Abcam), PTPN23 (ProteinTech), and horseradish peroxidase-conjugated goat anti-

423 Mouse IgG (BioRad) or HRP-donkey anti-Rabbit IgG (BioRad) and Western Clarity detection reagent

424 (BioRad). Apparent molecular mass was estimated using commercial protein standards (PageRulePlus, 
425 Thermo Scientific). Chemiluminescence was detected using a BioRad Chemi Doc imaging system and

426 analyzed using BioRad Image Lab v5.1 software.

427 Protein Biotinylation: HeLa P4.R5 cells were plated in $10 \mathrm{~cm}$ dishes at $3.2 \times 10^{6}$ cells per dish. The

428 following day, the cells were transfected with $12 \mu \mathrm{g}$ plasmid DNA using Lipofectamine 2000

429 (Invitrogen/Thermo Fisher), following manufacturer's guidelines. Biotinylation and protein harvest was

430 performed 24 hours later, following established protocol (41). The cells were incubated with $500 \mu \mathrm{M}$

431 biotinyl-tyramide in pre-warmed complete DMEM for 30 minutes at $37^{\circ} \mathrm{C}$. The biotinylation reaction

432 was catalyzed by addition of $1 \mathrm{mM}$ hydrogen peroxide to the culture media for 1 minute before

433 quenching three times with APEX2 quenching solution (10 mM sodium ascorbate, $5 \mathrm{mM}$ Trolox, and 10

$434 \mathrm{mM}$ sodium azide in $1 \mathrm{X}$ PBS). The cells were scraped from the dishes with the final quencher wash into

$43515 \mathrm{~mL}$ Falcon tubes and pelleted by centrifugation at $300 \times \mathrm{g}$ for 5 minutes at $4^{\circ} \mathrm{C}$. The cell pellets were

436 lysed in 1ml RIPA buffer containing quenching components and protease inhibitor cocktail (41) for 5

437 minutes on ice. The lysates were briefly vortexed and nuclei pelleted by centrifugation at $15,000 \times \mathrm{g}$ for

43810 minutes at $4^{\circ} \mathrm{C}$. Protein content from the supernate was measured by Bradford protein assay

439 (BioRad) and equal amounts incubated with streptavidin beads at $4^{\circ} \mathrm{C}$ overnight, while gently agitated.

440 The following day, the beads were washed 2x with RIPA lysis buffer, and 1x with $2 \mathrm{M}$ urea in $10 \mathrm{mM}$ Tris-

$441 \mathrm{HCl}(\mathrm{pH} 8)$. The beads were washed again with RIPA lysis buffer, $2 \times 1 \times$ PBS, and protein eluted from the

442 beads in excess Biotin (200 mM NaCl, $50 \mathrm{mM}$ Tris- $\mathrm{HCl}\left(\mathrm{pH}\right.$ 8), 2\% SDS, $1 \mathrm{mM} \mathrm{D-Biotin)}$ at $70^{\circ} \mathrm{C}$ for 30

443 minutes. An aliquot was stored for Western blot analysis, and remaining eluate processed for mass

444 spectrometric analysis.

445 Quantitative Mass Spectrometry: Quantitative MS analysis was performed as previously described (42)

446 in the Collaborative Center for Multiplexed Proteomics in the Department of Pharmacology and the

447 Skaggs School of Pharmacy and Pharmaceutical Sciences at UC San Diego. All quantitative mass 
spectrometry experiments were performed in biological duplicate. Protein disulfides were reduced with

(TCA) on ice for $10 \mathrm{~min}$. Precipitated proteins were pelleted by centrifugation at $14,000 \mathrm{rpm}$ for $5 \mathrm{~min}$, 
472 (42). The data from the proteomic experiments have been uploaded to ProteomeXchange (PXD023713)

473 through MassIVE (MSV000086733).

474 Global Arrayed Protein Stability Analysis (GAPSA): The GAPSA assay was performed as previously

475 described (28). cDNA clones for approximately 160 genes were isolated from the Human ORFeome V8.1

476 Collection (Broad Institute). cDNA concentrations were normalized to $10 \mathrm{ng} / \mu \mathrm{L}$ prior to spotting in poly-

477 D-lysine-coated 384 well plates. 20 ng cDNA encoding Vpu-FLAG, Vpu-S52,56N-FLAG or LacZ-FLAG

478 control diluted in Opti-MEM was added to each well. Fugene6 (Promega) transfection reagent was

479 added and incubated for 25 minutes at room temperature. $20 \mu \mathrm{L}$ DMEM containing $6 \times 10^{4}$ HEK293T

480 cells was added to each well and subsequently incubated for 48 hours at $37^{\circ} \mathrm{C}, 5 \% \mathrm{CO}_{2}$. The cells were

481 stained using an automated protocol. First, the plates were washed with PBS and cells fixed in 8\%

482 paraformaldehyde for 1 hour at room temperature. The cells were then washed with PBS, and

483 permeabilized with $0.5 \%$ Triton X-100 in PBS for 10 minutes at room temperature. The cells were then

484 washed with PBS and incubated with 6\% BSA in PBS for 1 hour at room temperature to block non-

485 specific antigen binding. The cells were then washed with PBS and incubated with mouse anti-V5 and

486 rabbit anti-FLAG antibodies (1:250) in BSA-PBS for 1 hour at room temperature. The cells then were

487 washed with PBS and incubated with goat anti-mouse Alexa 488, and goat anti-rabbit Alexa 568 (1:250

488 dilution) in BSA-PBS for 1 hour at room temperature. Nuclei were then stained with $2 \mu \mathrm{g} / \mathrm{mL}$ DAPI. The

489 plates were imaged using the Opera QEHS High-Content Imaging System. The image output was

490 analyzed using the Acapella High-Content Image Analysis Software (PerkinElmer) using a custom script.

491 Data analysis was done as previously described (28).

492 Data presentation and statistics. Figures were prepared using Adobe Creative Suite CS3;

493 immunofluorescence images were adjusted using Adobe Photoshop and figures prepared using Adobe

494 Illustrator software. Statistical analyses were performed using Graphpad Prism v5. Mass spectrometry 
data was analyzed using Microsoft excel 2016 and R Studio (R v.4.0.3). Significance for proteomics data

using Morpheus matrix visualization software (Broad institute,

499 https://software.broadinstitute.org/morpheus); data were sorted by k-means clustering, the optimal

500 number of gene clusters was determined by elbow estimation method. Gene Ontology (GO) analysis was

501 performed using the Database for Annotation, Visualization and Integrated Discovery (DAVID) v6.8 (43,

502 44). Network analysis of protein subset k-means cluster 6 was performed using the STRING app for

503 Cytoscape $(v 3.7 .0)(45,46)$.

\section{Acknowledgements}

505 We thank Klaus Strebel for the original codon-optimized VpHu construct and pNL4-3AVpu; Alice Ting for 506 APEX-related constructs; the NIH AIDS Reagent Program; and The Pendleton Charitable Trust. The work 507 was supported by NIH grants R37AI081668 to JCG, R01 Al124843 and R01 Al127302 to SKC, and in part

508 by UC San Diego Center for AIDS Research Developmental Awards to CAS, LP, and DG; an NIH-funded 509 program (P30 Al036214). SL was supported by a research fellowship of the Deutsche

510 Forschungsgemeinschaft (DFG) (Grant reference number 404687549). JMW was supported by NIH T32

511 GM007752 and T32 AR064194, JL was supported by NIH K12 GM06852.

\section{$512 \quad$ Competing interests}

513 The authors have no financial or non-financial competing interests.

\section{$514 \quad$ Supplementary Materials}

515 Figure S1: Juxtanuclear endosomal distortion in cells expressing Vpu-S52,56N-APEX2. 
bioRxiv preprint doi: https://doi org/10.1101/2021.02 22.432252. this version posted February 22 2021. The copyright holder for this preprint

(which was not certified by peer review) is the author/funder. This article is a US Government work. It is not subject to copyright under 17 USC 105 and is also made available for use under a CCO license.

517 Figure S3: Pair-wise comparison of proximity-ome of Vpu-APEX2 compared to the Mito-APEX2.

518 Figure S4: Immunofluorescence microscopy of Vpu-FLAG and candidate cofactor SNX3

519 Table S1: Mass Spectrometry Experiment 1: Comparison of Mito Matrix and Vpu WT and mutants

520 Table S2: Mass Spectrometry Experiment 2 and 3: Comparison of Vpu WT and mutants

521 Table S3: Gene Ontology network analysis of differentially-enriched Vpu-proximal protein subsets 


\section{References}

523 1. Van Damme N, Goff D, Katsura C, Jorgenson RL, Mitchell R, Johnson MC, et al. The interferon-

524 induced protein BST-2 restricts HIV-1 release and is downregulated from the cell surface by the viral Vpu

525 protein. Cell host \& microbe. 2008;3(4):245-52.

$5262 . \quad$ Neil SJ, Zang T, Bieniasz PD. Tetherin inhibits retrovirus release and is antagonized by HIV-1 Vpu.

527 Nature. 2008;451(7177):425-30.

528 3. Lambele M, Koppensteiner H, Symeonides M, Roy NH, Chan J, Schindler M, et al. Vpu is the main 529 determinant for tetraspanin downregulation in HIV-1-infected cells. J Virol. 2015;89(6):3247-55.

530 4. Willey RL, Maldarelli F, Martin MA, Strebel K. Human immunodeficiency virus type 1 Vpu protein

531 induces rapid degradation of CD4. Journal of virology. 1992;66(12):7193-200.

532 5. Shah AH, Sowrirajan B, Davis ZB, Ward JP, Campbell EM, Planelles V, et al. Degranulation of 533 natural killer cells following interaction with HIV-1-infected cells is hindered by downmodulation of NTB-

534 A by Vpu. Cell host \& microbe. 2010;8(5):397-409.

535 6. Ramirez PW, Famiglietti M, Sowrirajan B, DePaula-Silva AB, Rodesch C, Barker E, et al.

536 Downmodulation of CCR7 by HIV-1 Vpu results in impaired migration and chemotactic signaling within

537 CD4(+) T cells. Cell reports. 2014;7(6):2019-30.

538 7. Apps R, Del Prete GQ, Chatterjee P, Lara A, Brumme ZL, Brockman MA, et al. HIV-1 Vpu Mediates

539 HLA-C Downregulation. Cell Host Microbe. 2016;19(5):686-95.

540 8. Margottin F, Bour SP, Durand H, Selig L, Benichou S, Richard V, et al. A novel human WD protein,

541 h-beta TrCp, that interacts with HIV-1 Vpu connects CD4 to the ER degradation pathway through an F-

542 box motif. Molecular cell. 1998;1(4):565-74. 
543 9. Magadan JG, Perez-Victoria FJ, Sougrat R, Ye Y, Strebel K, Bonifacino JS. Multilayered mechanism

544 of CD4 downregulation by HIV-1 Vpu involving distinct ER retention and ERAD targeting steps. PLoS

545 pathogens. 2010;6(4):e1000869.

546 10. Mitchell RS, Katsura C, Skasko MA, Fitzpatrick K, Lau D, Ruiz A, et al. Vpu antagonizes BST-2-

547 mediated restriction of HIV-1 release via beta-TrCP and endo-lysosomal trafficking. PLoS pathogens.

$548 \quad 2009 ; 5(5): \mathrm{e} 1000450$.

549 11. Douglas JL, Viswanathan K, McCarroll MN, Gustin JK, Fruh K, Moses AV. Vpu directs the

550 degradation of the human immunodeficiency virus restriction factor BST-2/Tetherin via a \{beta\}TrCP-

551 dependent mechanism. Journal of virology. 2009;83(16):7931-47.

552 12. Schmidt S, Fritz JV, Bitzegeio J, Fackler OT, Keppler OT. HIV-1 Vpu blocks recycling and

553 biosynthetic transport of the intrinsic immunity factor CD317/tetherin to overcome the virion release

554 restriction. mBio. 2011;2(3):e00036-11.

555 13. Dube M, Roy BB, Guiot-Guillain P, Binette J, Mercier J, Chiasson A, et al. Antagonism of tetherin 556 restriction of HIV-1 release by Vpu involves binding and sequestration of the restriction factor in a 557 perinuclear compartment. PLoS pathogens. 2010;6(4):e1000856.

558 14. Lau D, Kwan W, Guatelli J. Role of the endocytic pathway in the counteraction of BST-2 by

559 human lentiviral pathogens. Journal of virology. 2011;85(19):9834-46.

560 15. Kueck T, Neil SJ. A cytoplasmic tail determinant in HIV-1 Vpu mediates targeting of tetherin for 561 endosomal degradation and counteracts interferon-induced restriction. PLoS pathogens.

$562 \quad 2012 ; 8(3): \mathrm{e} 1002609$. 

dependent Interactions with Clathrin Adaptors. Traffic. 2017. mediated BST2 antagonism via hijacking of the clathrin adaptor protein complex 1. eLife.

$567 \quad 2014 ; 3: \mathrm{e} 02362$.

568

569

570

19. Janvier K, Pelchen-Matthews A, Renaud JB, Caillet M, Marsh M, Berlioz-Torrent C. The ESCRT-0

572 component HRS is required for HIV-1 Vpu-mediated BST-2/tetherin down-regulation. PLoS Pathog.

573 2011;7(2):e1001265. 20. Van Damme N, Guatelli J. HIV-1 Vpu inhibits accumulation of the envelope glycoprotein within 575 clathrin-coated, Gag-containing endosomes. Cell Microbiol. 2008;10(5):1040-57.

577 pericentriolar recycling endosome plays a key role in Vpu-mediated enhancement of HIV-1 particle 578 release. Traffic. 2006;7(3):298-307.

579 22. Dube M, Roy BB, Guiot-Guillain P, Mercier J, Binette J, Leung G, et al. Suppression of Tetherinrestricting activity upon human immunodeficiency virus type 1 particle release correlates with

581 localization of Vpu in the trans-Golgi network. Journal of virology. 2009;83(9):4574-90.

23. Lam SS, Martell JD, Kamer KJ, Deerinck TJ, Ellisman MH, Mootha VK, et al. Directed evolution of 
584 24. Martell JD, Deerinck TJ, Sancak Y, Poulos TL, Mootha VK, Sosinsky GE, et al. Engineered

585 ascorbate peroxidase as a genetically encoded reporter for electron microscopy. Nature biotechnology.

$586 \quad 2012 ; 30(11): 1143-8$

587 25. Hauser H, Lopez LA, Yang SJ, Oldenburg JE, Exline CM, Guatelli JC, et al. HIV-1 Vpu and HIV-2 Env

588 counteract BST-2/tetherin by sequestration in a perinuclear compartment. Retrovirology. 2010;7:51.

589 26. Skasko M, Tokarev A, Chen CC, Fischer WB, Pillai SK, Guatelli J. BST-2 is rapidly down-regulated

590 from the cell surface by the HIV-1 protein Vpu: evidence for a post-ER mechanism of Vpu-action.

591 Virology. 2011;411(1):65-77.

592 27. Skasko M, Wang Y, Tian Y, Tokarev A, Munguia J, Ruiz A, et al. HIV-1 Vpu protein antagonizes

593 innate restriction factor BST-2 via lipid-embedded helix-helix interactions. The Journal of biological

594 chemistry. 2012;287(1):58-67.

595 28. Jain P, Boso G, Langer S, Soonthornvacharin S, De Jesus PD, Nguyen Q, et al. Large-Scale Arrayed

596 Analysis of Protein Degradation Reveals Cellular Targets for HIV-1 Vpu. Cell reports. 2018;22(9):2493-

597503.

598 29. Doyotte A, Mironov A, McKenzie E, Woodman P. The Bro1-related protein HD-PTP/PTPN23 is

599 required for endosomal cargo sorting and multivesicular body morphogenesis. Proc Natl Acad Sci U S A.

$600 \quad 2008 ; 105(17): 6308-13$.

601 30. Matheson NJ, Sumner J, Wals K, Rapiteanu R, Weekes MP, Vigan R, et al. Cell Surface Proteomic

602 Map of HIV Infection Reveals Antagonism of Amino Acid Metabolism by Vpu and Nef. Cell host \&

603 microbe. 2015;18(4):409-23. 
604 31. Jager S, Cimermancic P, Gulbahce N, Johnson JR, McGovern KE, Clarke SC, et al. Global landscape

605 of HIV-human protein complexes. Nature. 2011;481(7381):365-70.

606 32. Ali N, Zhang L, Taylor S, Mironov A, Urbe S, Woodman P. Recruitment of UBPY and ESCRT

607 exchange drive HD-PTP-dependent sorting of EGFR to the MVB. Curr Biol. 2013;23(6):453-61.

608 33. Raiborg C, Bache KG, Gillooly DJ, Madshus IH, Stang E, Stenmark H. Hrs sorts ubiquitinated

609 proteins into clathrin-coated microdomains of early endosomes. Nat Cell Biol. 2002;4(5):394-8.

610 34. Barlowe C, Schekman R. SEC12 encodes a guanine-nucleotide-exchange factor essential for

611 transport vesicle budding from the ER. Nature. 1993;365(6444):347-9.

612 35. Dube M, Paquay C, Roy BB, Bego MG, Mercier J, Cohen EA. HIV-1 Vpu antagonizes BST-2 by

613 interfering mainly with the trafficking of newly synthesized BST-2 to the cell surface. Traffic.

$614 \quad 2011 ; 12(12): 1714-29$.

615 36. Charneau P, Mirambeau G, Roux P, Paulous S, Buc H, Clavel F. HIV-1 reverse transcription. A

616 termination step at the center of the genome. Journal of molecular biology. 1994;241(5):651-62.

617 37. Greenberg ME, Bronson S, Lock M, Neumann M, Pavlakis GN, Skowronski J. Co-localization of

618 HIV-1 Nef with the AP-2 adaptor protein complex correlates with Nef-induced CD4 down-regulation. The

619 EMBO journal. 1997;16(23):6964-76.

620 38. Yang X, Boehm JS, Yang X, Salehi-Ashtiani K, Hao T, Shen Y, et al. A public genome-scale lentiviral 621 expression library of human ORFs. Nature methods. 2011;8(8):659-61.

622 39. Adachi A, Gendelman HE, Koenig S, Folks T, Willey R, Rabson A, et al. Production of acquired

623 immunodeficiency syndrome-associated retrovirus in human and nonhuman cells transfected with an

624 infectious molecular clone. Journal of virology. 1986;59(2):284-91. 
625 40. Strebel K, Klimkait T, Martin MA. A novel gene of HIV-1, vpu, and its 16-kilodalton product.

626 Science. 1988;241(4870):1221-3.

627 41. Hung V, Udeshi ND, Lam SS, Loh KH, Cox KJ, Pedram K, et al. Spatially resolved proteomic

628 mapping in living cells with the engineered peroxidase APEX2. Nature protocols. 2016;11(3):456-75.

629 42. Lapek JD, Jr., Lewinski MK, Wozniak JM, Guatelli J, Gonzalez DJ. Quantitative Temporal Viromics

630 of an Inducible HIV-1 Model Yields Insight to Global Host Targets and Phospho-Dynamics Associated

631 with Protein Vpr. Mol Cell Proteomics. 2017;16(8):1447-61.

632 43. Huang da W, Sherman BT, Lempicki RA. Systematic and integrative analysis of large gene lists

633 using DAVID bioinformatics resources. Nature protocols. 2009;4(1):44-57.

634 44. Huang da W, Sherman BT, Lempicki RA. Bioinformatics enrichment tools: paths toward the 635 comprehensive functional analysis of large gene lists. Nucleic acids research. 2009;37(1):1-13.

636 45. Shannon P, Markiel A, Ozier O, Baliga NS, Wang JT, Ramage D, et al. Cytoscape: a software

637 environment for integrated models of biomolecular interaction networks. Genome Res.

$638 \quad 2003 ; 13(11): 2498-504$.

639 46. Szklarczyk D, Gable AL, Lyon D, Junge A, Wyder S, Huerta-Cepas J, et al. STRING v11: protein-

640 protein association networks with increased coverage, supporting functional discovery in genome-wide

641 experimental datasets. Nucleic acids research. 2019;47(D1):D607-D13. 
A
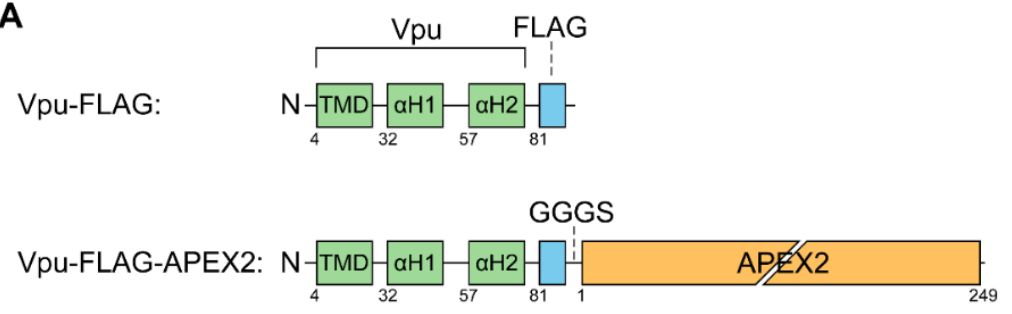

B

C
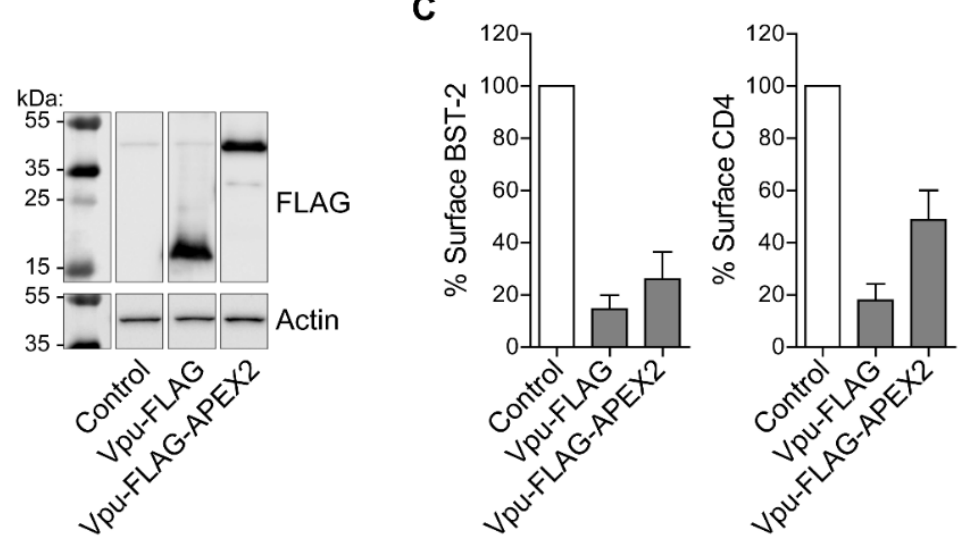

644 Figure 1. Vpu-APEX2 fusion protein design and activity. (A) A schematic representation of C-terminally tagged Vpu constructs; Vpu-FLAG and Vpu-FLAG-APEX2. A GGGS linker lies between the FLAG epitope and APEX2. (B) HeLa P4.R5 cells were transfected with Vpu constructs bearing C-terminal FLAG or FLAG

647 and APEX2. Protein expression was analysed by western blot. (C) Cell-surface levels of BST-2 and CD4 were measured in the presence of Vpu-FLAG or Vpu-FLAG-APEX2 by flow cytometry. Surface levels of BST-2 and CD4 on cells expressing FLAG- or FLAG-APEX2-tagged Vpu was expressed as the \% of control cells not expressing Vpu. Error bars represent standard deviation of $n=3$ experiments. 
bioRxiv preprint doi: https://doi.org/10.1101/2021.02.22.432252; this version posted February 22. 2021. The copyright holder for this preprint (which was not certified by peer review) is the author/funder. This article is a US Government work. It is not subject to copyright under 17 USC 105 and is also made available for use under a CCO license.

A
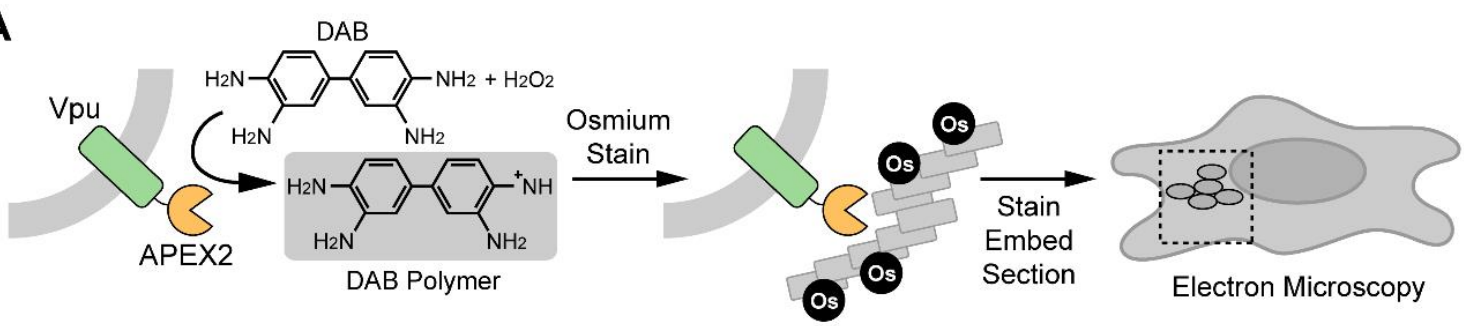

Electron Microscopy

B
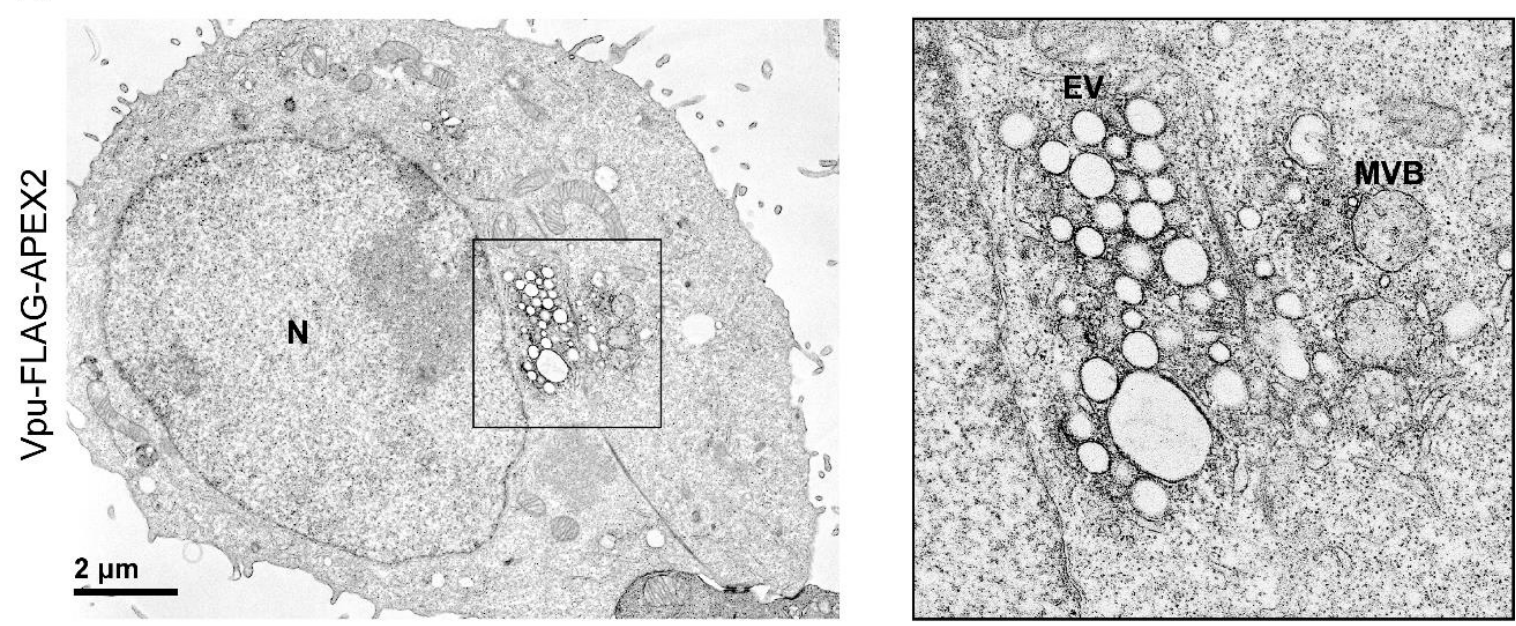

C

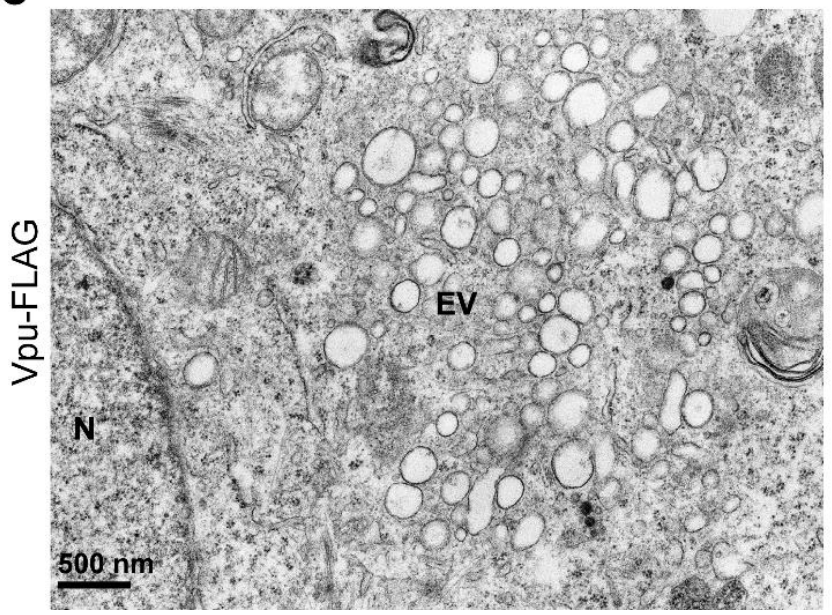

D

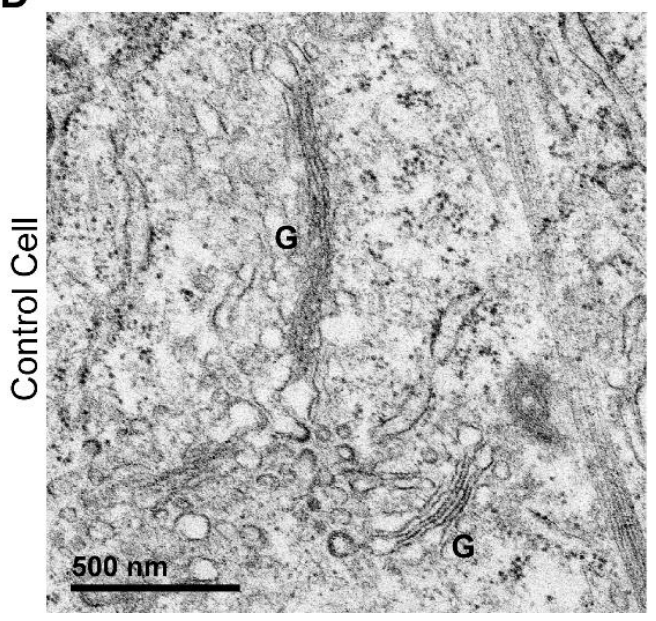

Figure 2. Vpu-APEX2 localizes to enlarged juxtanuclear endosomes and the limiting membranes of

multi-vesicular bodies. (A) Schematic depicting APEX2 staining protocol for visualization by electron

654 microscopy. (B) HeLa P4.R5 cells were transfected with the codon-optimized Vpu constructs bearing a

655 C-terminal APEX2 tag. 24 hours later the cells were fixed before APEX2-dependent polymerization of 
657 imaged by transmission electron microscopy (TEM). Left panel: Cells expressing Vpu-APEX2 contain

658 juxtanuclear accumulations of osmium-highlighted enlarged vesicles (EV) likely derived from the Golgi

659 and endosomes. Right panel: a higher magnification image of the juxtanuclear region of the cell shown

660 at left. The limiting membranes of vesicles resembling multivesicular bodies (MVB) are highlighted by

661 osmium. (C) Cells expressing Vpu (without an APEX2 tag) also contain enlarged juxtanuclear vesicles. (D)

662 A control image showing Golgi (G) stacks in non-transfected HeLa P4.R5 cells. 
bioRxiv preprint doi: https://doi.org/10.1101/2021.02.22 432252; this version posted February 22, 2021. The copyright holder for this preprint (which was not certified by peer review) is the author/funder. This article is a US Government work. It is not subject to copyright under 17 USC 105 and is also made available for use under a CCO license.

A

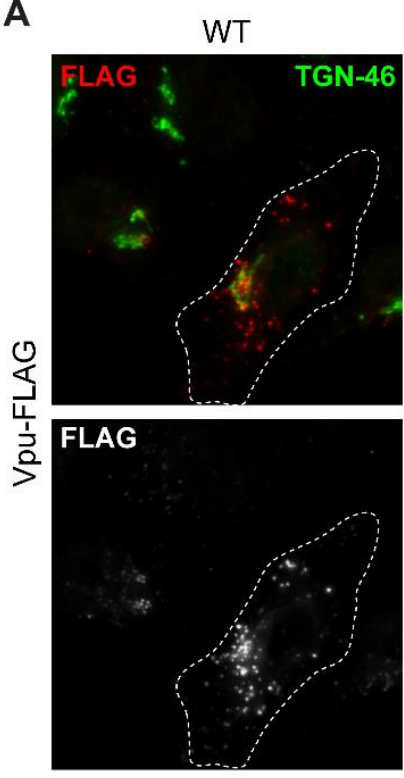

B

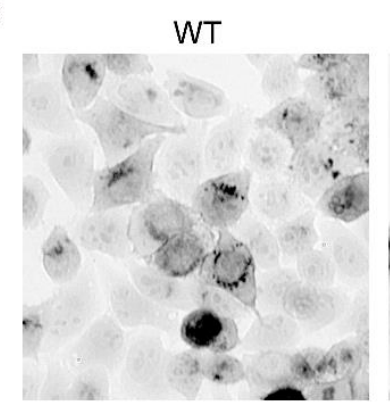

C

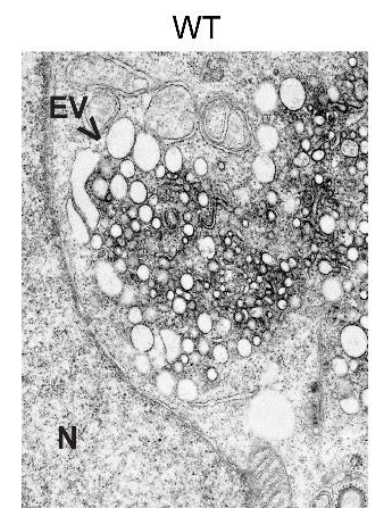

$\mathrm{A} 18 \mathrm{H}$
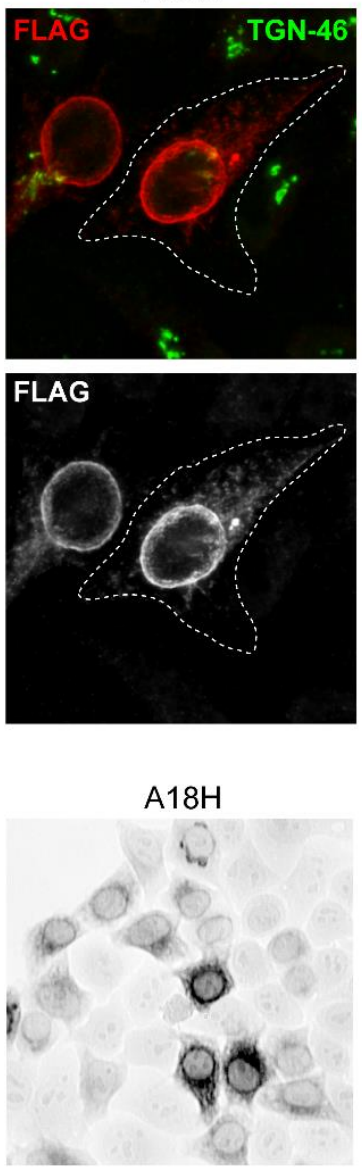

$\mathrm{A} 18 \mathrm{H}$

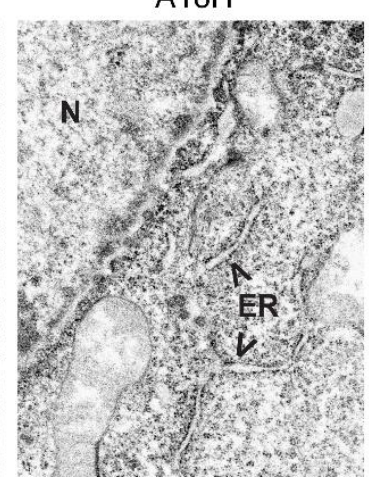

AAA/F
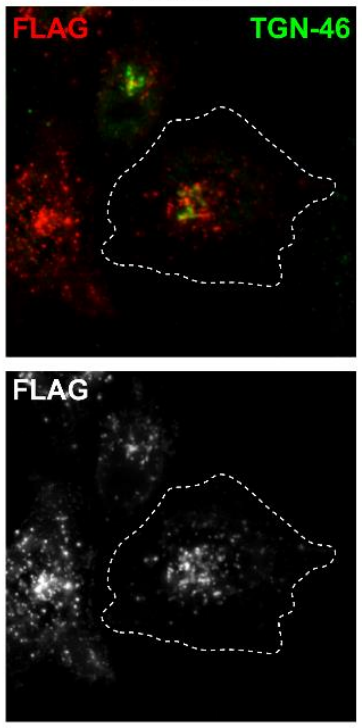

AAA/F

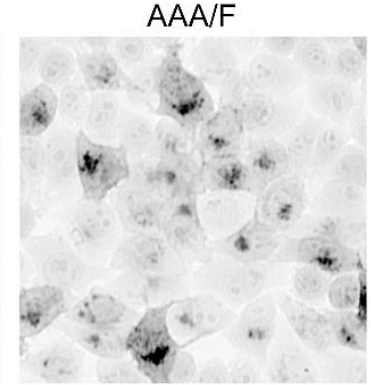

AAA/F

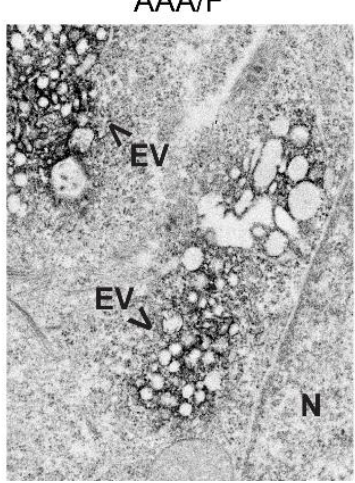

$\mathrm{S} 52,56 \mathrm{~N}$
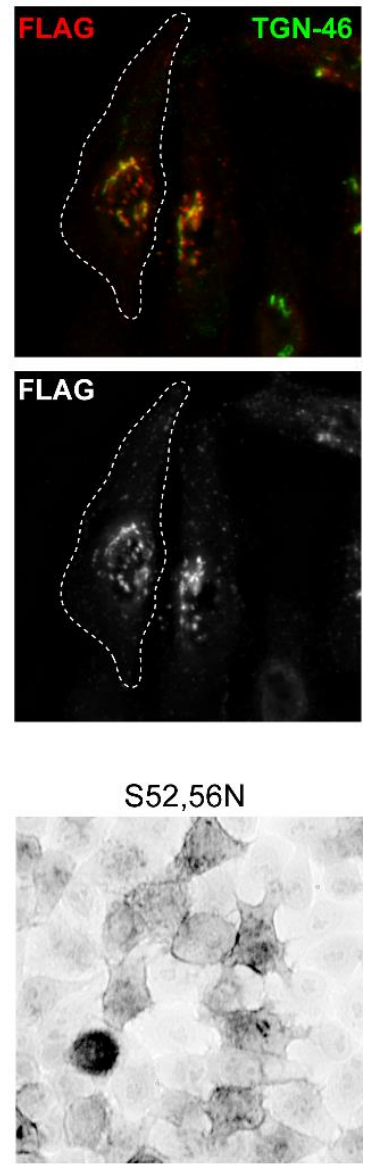

$\mathrm{S} 52,56 \mathrm{~N}$

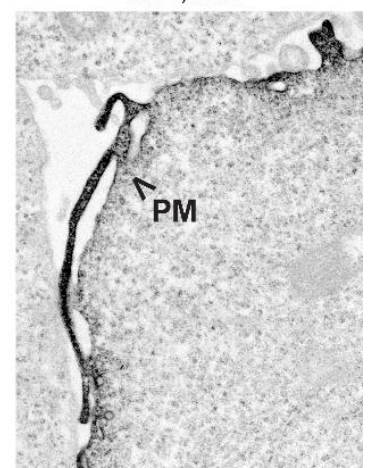

664 Figure 3. Light and electron microscopic distributions of Vpu-APEX2 and mutants A18H, AAA/F, and

S52,56N. (A) HeLa P4.R5 cells were transfected to express Vpu-FLAG (A), either wild type or encoding

666 the mutations $A 18 \mathrm{H}, \mathrm{AAA} / \mathrm{F}$, or $\mathrm{S} 52,56 \mathrm{~N}$. The cells were fixed and stained for FLAG (shown in red) and

667 TGN-46 (shown in green). (B) HeLa P4.R5 cells were transfected to express Vpu WT and the indicated 
bioRxiv preprint doi: https://doi.org/10.1101/2021.02 22 432252 this version posted February 22.2021 . The copyright holder for this preprint

(which was not certified by peer review) is the author/funder. This article is a US Government work. It is not subject to copyright under 17 USC 105 and is also made available for use under a CCO license.

668 mutants tagged with APEX2; 24 hours later the cells were reacted with DAB in the presence of hydrogen

669 peroxide. The cells were stained and osmiophilic DAB polymer visualized in whole-cells by brightfield

670 microscopy. (C) Thin-section electron microscopy of cells expressing Vpu WT-, A18H-, AAA/F-, or

671 S52,56N-APEX2. Arrows indicate concentrations of osmiophilic polymer stain. $\mathrm{N}=$ nucleus, $E R=$

672 endoplasmic reticulum, $\mathrm{PM}=$ plasma membrane. 
A

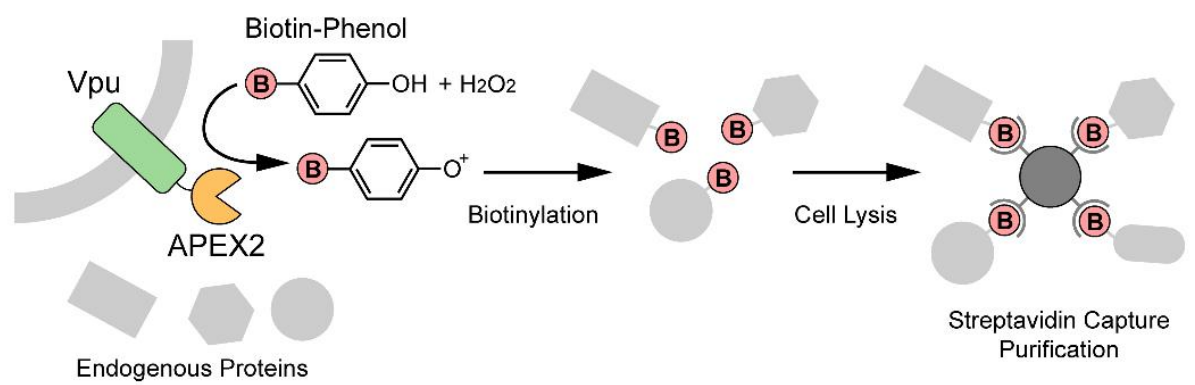

B
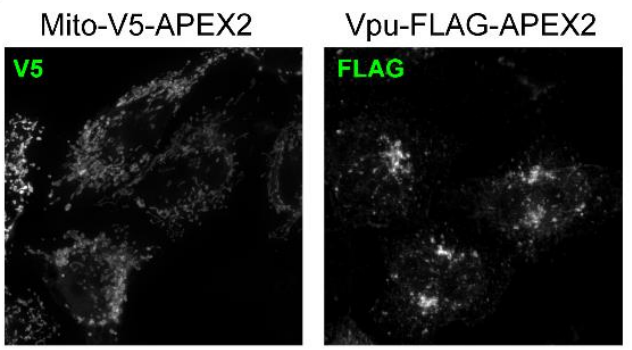

C
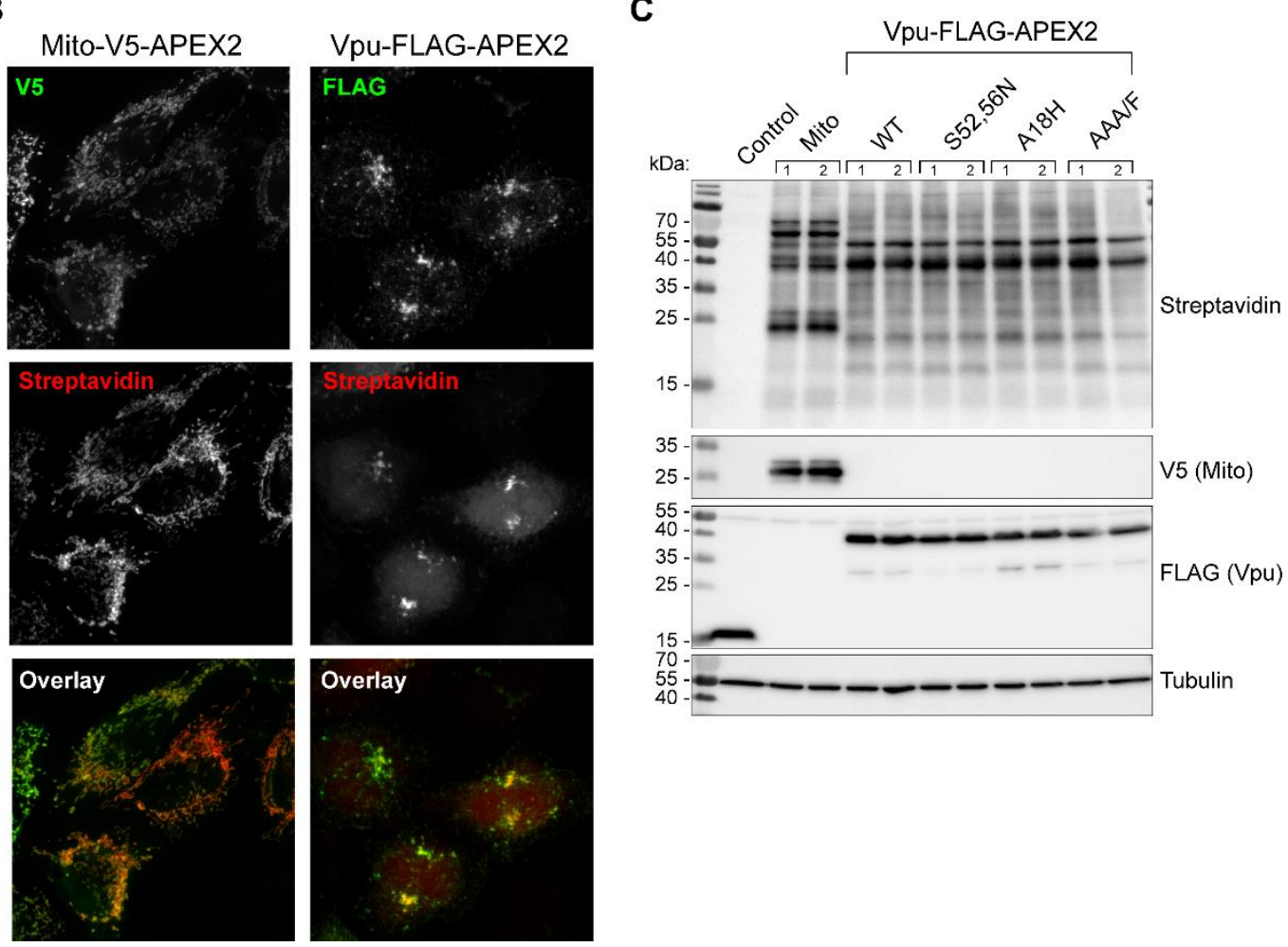

674 Figure 4. Biotinylation of Vpu-APEX2 proximal proteins. (A) Schematic representation of APEX2-

675 mediated biotinylation reaction; in the presence of hydrogen peroxide and biotin-phenol, APEX2

676 catalyses biotinylation of nearby proteins, which can be captured by streptavidin. (B) Detection of

677 biotinylated proteins by immunofluorescent-streptavidin. HeLa P4.R5 cells expressing Mito-V5-APEX2 or

678 Vpu-FLAG-APEX2 were incubated with biotin phenol and hydrogen peroxide for 1 minute before

679 quenching, fixation, and staining with streptavidin-alexa594 (red) and anti-V5 or anti-FLAG (green). (C)

680 Biotinylation pattern of protein species visualized by western blot; HeLa P4.R5 cells transfected to

681 express Mito-V5-APEX or WT Vpu-, S52,56N-, A18H-, or AAA/F-FLAG-APEX2 were incubated with biotin- 
bioRxiv preprint doi: https://doi.org/10.1101/2021.02.22.432252; this version posted February 22, 2021. The copyright holder for this preprint

(which was not certified by peer review) is the author/funder. This article is a US Government work. It is not subject to copyright under 17 USC 105 and is also made available for use under a CCO license.

682 phenol, lysed and proteins separated by SDS-PAGE and western blot. Biotinylated proteins were

683 detected using streptavidin-HRP. The control cells were transfected to express Vpu-FLAG only;

684 streptavidin staining is absent in the absence of APEX2. 
A
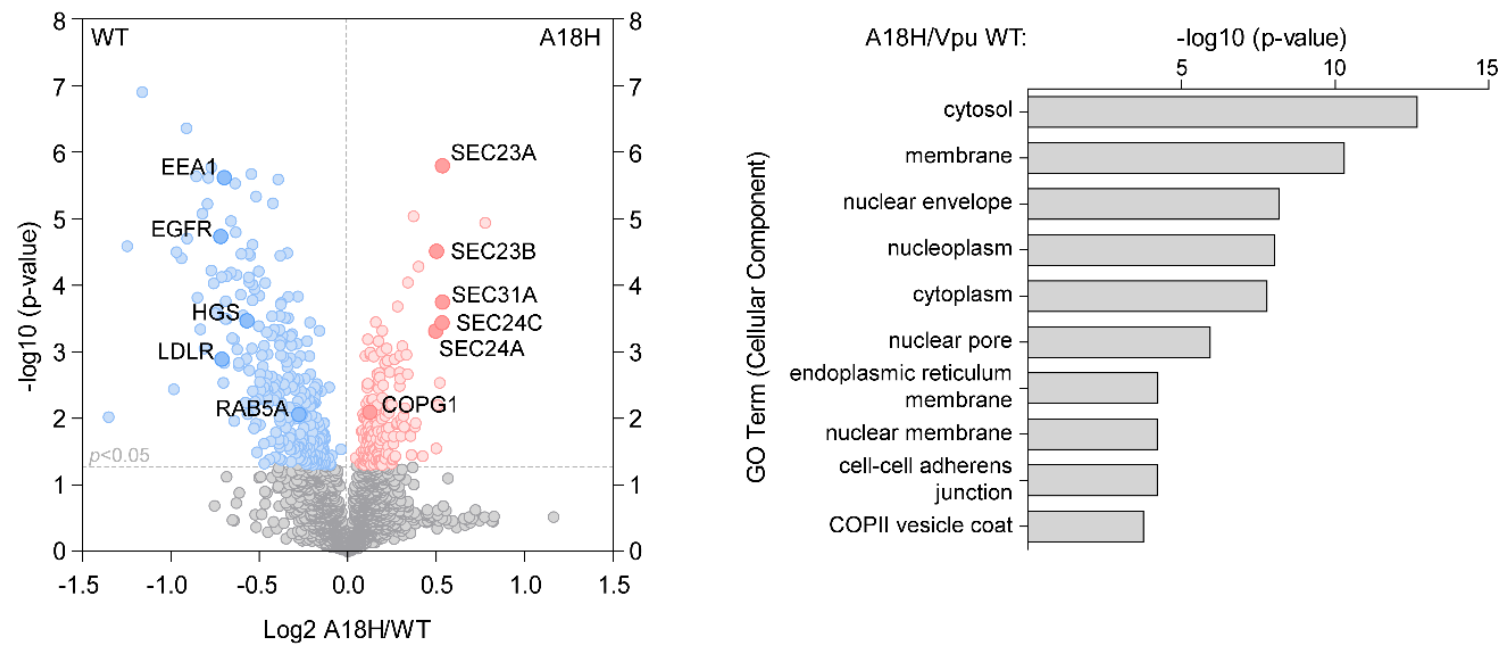

B
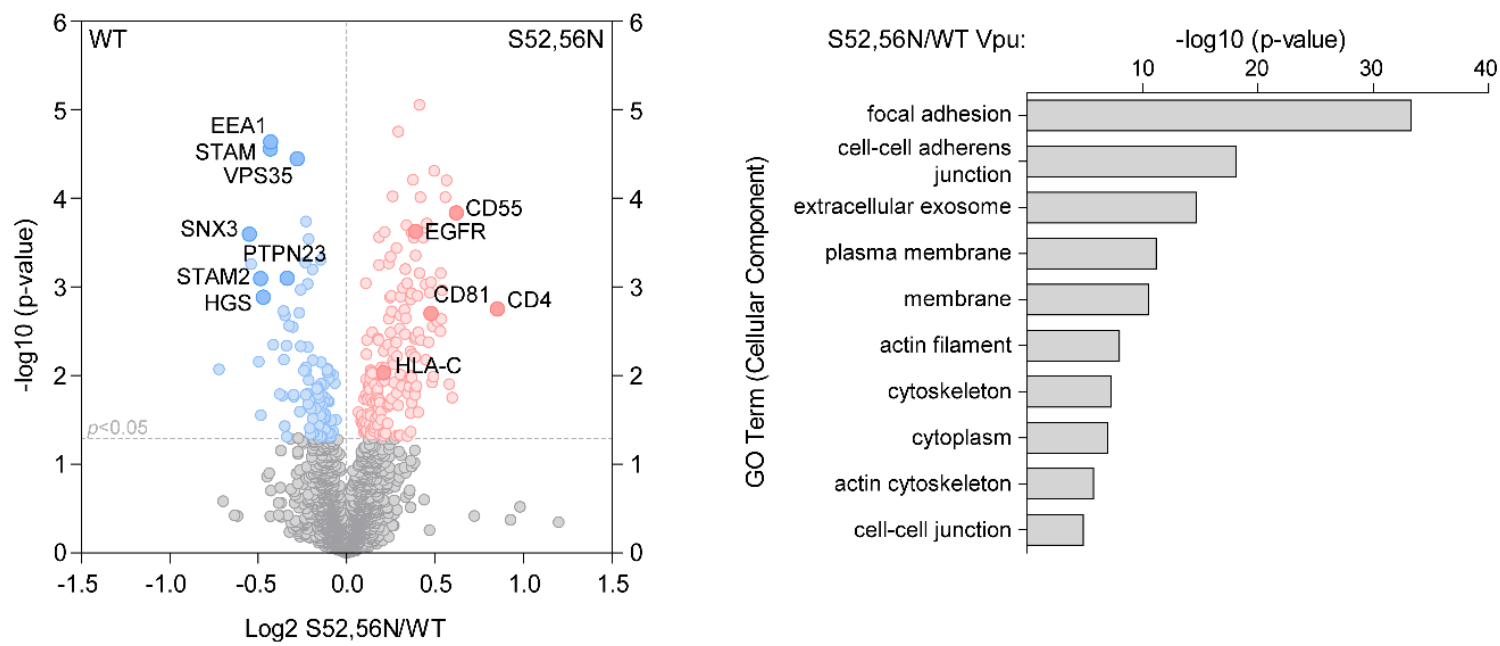

Figure 5. Pair-wise comparisons of the proximity-omes of wild type Vpu relative to the ER-retained

Vpu-A18H and the plasma-membrane-enriched Vpu-S52,56N mutants. HeLa P4.R5 cells were 
694 for proteins associated with plasma and endosomal membranes (labeled). (B) The S52,56N mutant was

695 enriched in proteins derived from the plasma membrane (labeled), while WT Vpu was again enriched in

696 endosomal sorting proteins (labeled). Proteins enriched by the S52,56N mutation included the known

697 targets CD4, CD81, and HLA-C, and possible targets EGFR and CD55. For both (A) and (B), the x-axis

698 shows log2 fold change of proteins enriched by mutant/WT Vpu and the $y$-axis -log10 of $p$-value derived

699 from Student's $t$-test. The 10 most highly enriched gene ontology (cellular component) terms are shown

700 on the right of each volcano plot, corresponding to significantly enriched proteins proximal to the

701 mutants; $p$-value derived from Bonferroni test. 
A

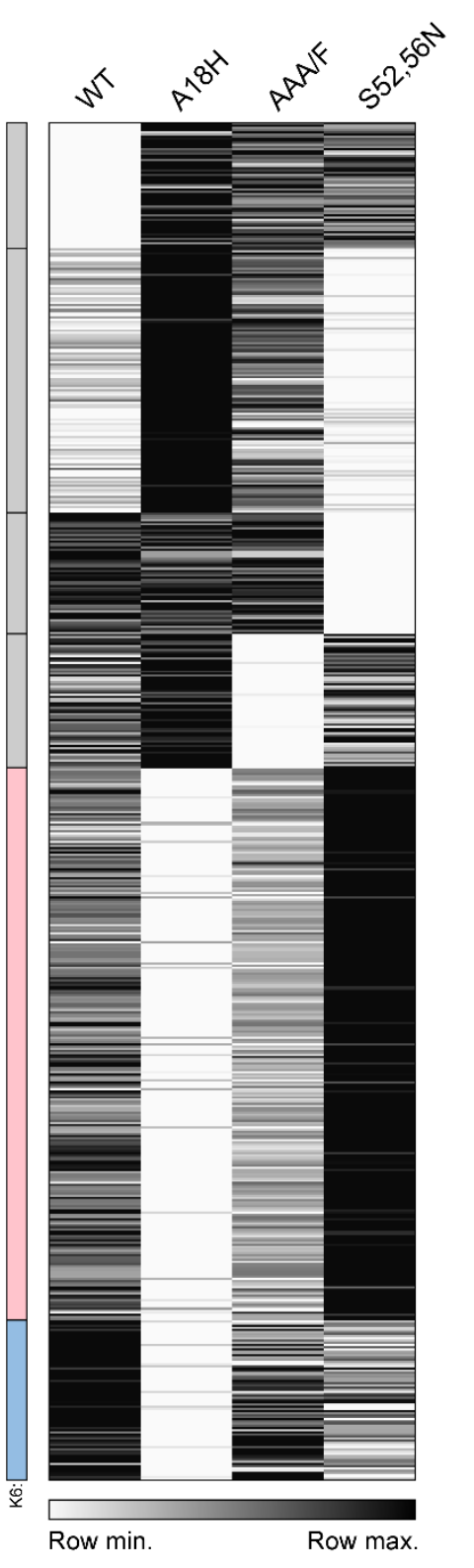

B

Cluster 5:
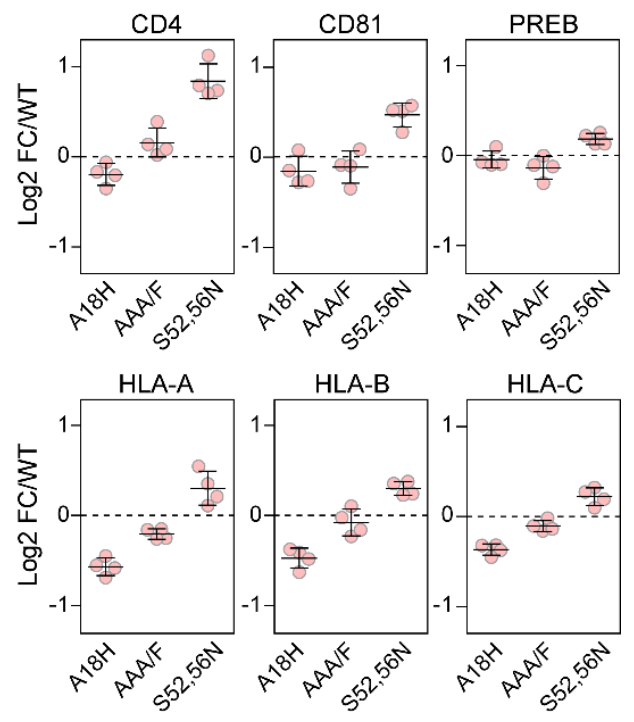

Cluster 6:
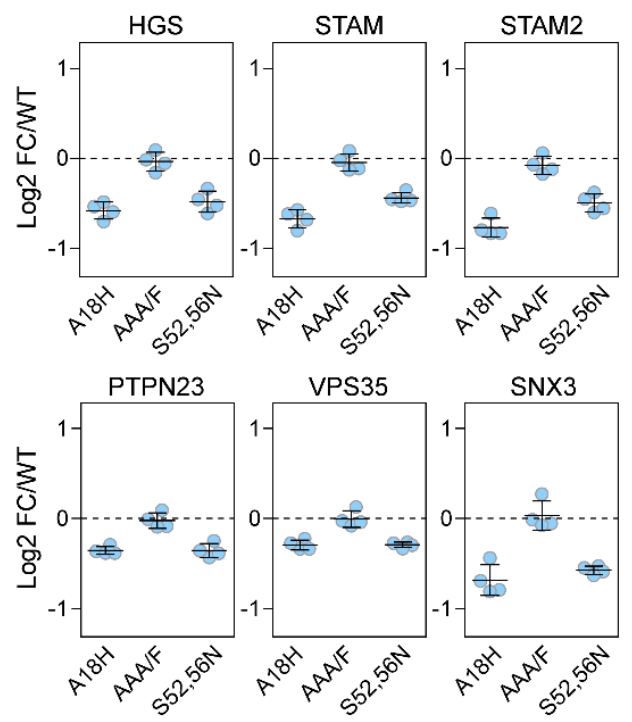

Figure 6. Heat map and k-means clustering of proteins (384) for which any Vpu-mutant was

significantly different from wild type. (A) Heatmap of the relative abundance of proteins measured in

Vpu-APEX2 WT and mutant samples. The heatmap was sorted into 6 clusters by k-means clustering

analysis; the cluster profile is shown on the left. Data are presented as the fold change in protein

abundance relative to WT. (B) Cluster 5 contains known and potential targets of Vpu, including CD4,

CD81, and HLA-C. k-means cluster 6 contains potential serine-dependent cofactors of Vpu, including 
bioRxiv preprint doi: https://doi.org/10.1101/2021.02.22.432252; this version posted February 22, 2021. The copyright holder for this preprint

(which was not certified by peer review) is the author/funder. This article is a US Government work. It is not subject to copyright under 17 USC 105 and is also made available for use under a CCO license. independent experiments. 
A

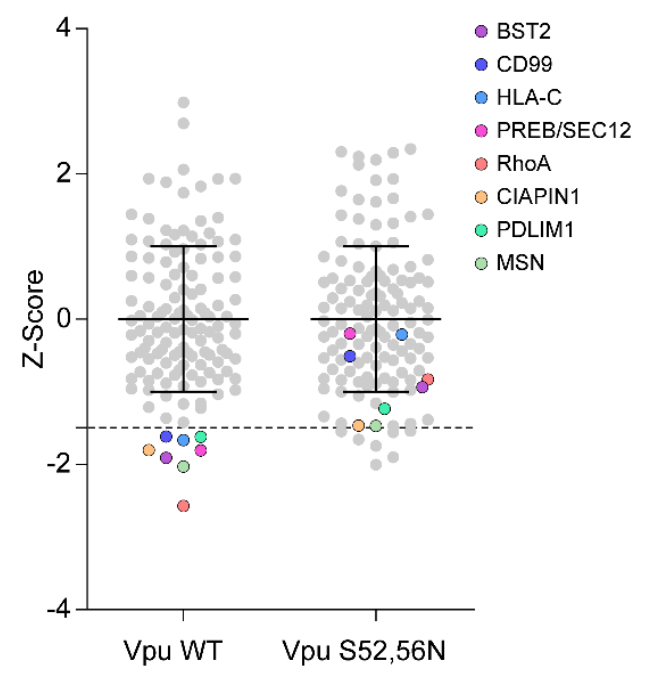

B
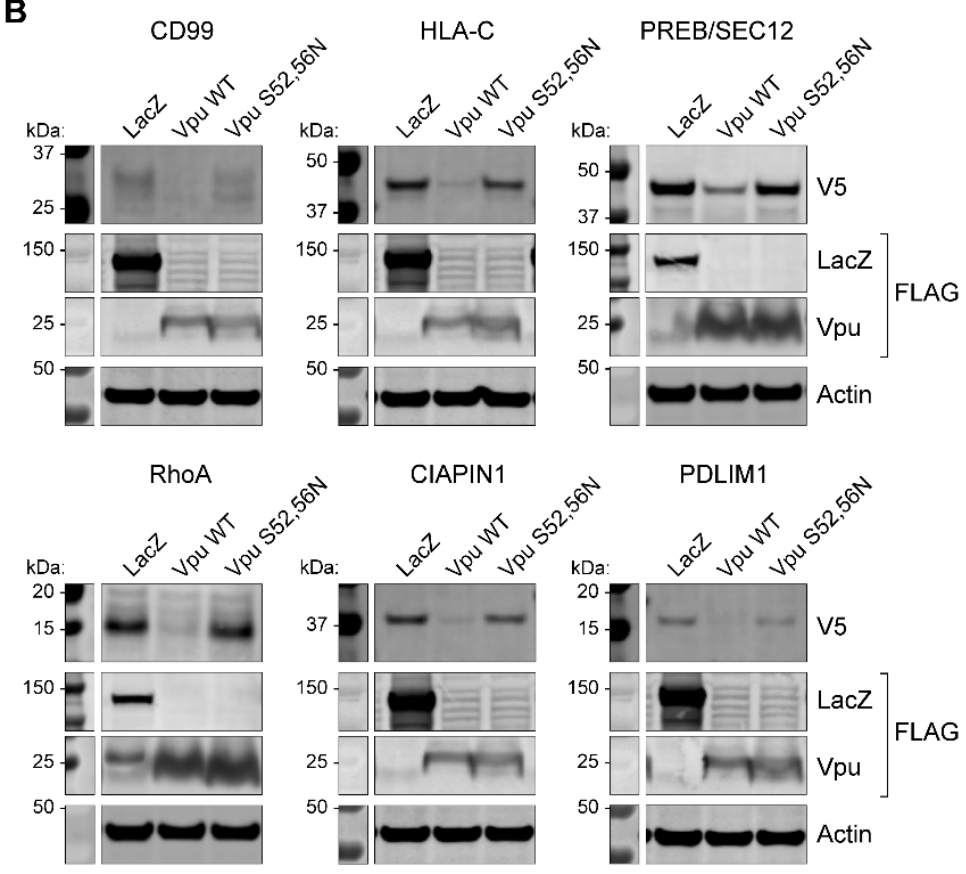

712 Figure 7. Proteins enriched in the proximity-ome of Vpu-S52,56N relative to wild type: Vpu-mediated

713 and serine-dependent decreases in steady-state expression. (A) HEK293T cells were co-transfected

714 with target cDNAs and plasmids expressing WT Vpu or Vpu-S52,56N. Levels of proteins were measured

71548 hours later by immunofluorescent staining and automated quantification of fluorescence. Candidate

716 Vpu targets whose expression is significantly decreased by Vpu are colored. (B) Vpu-mediated

717 degradation of putative target proteins was tested by western blotting of exogenously-expressed cDNA targets. 
A

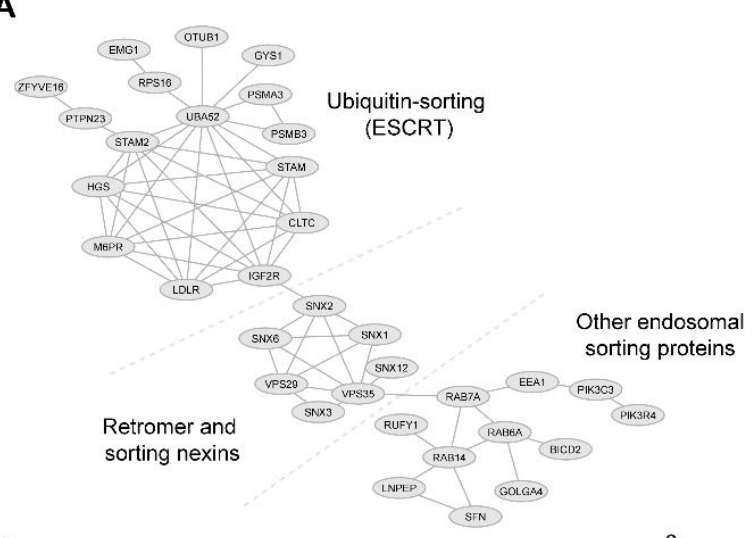

C
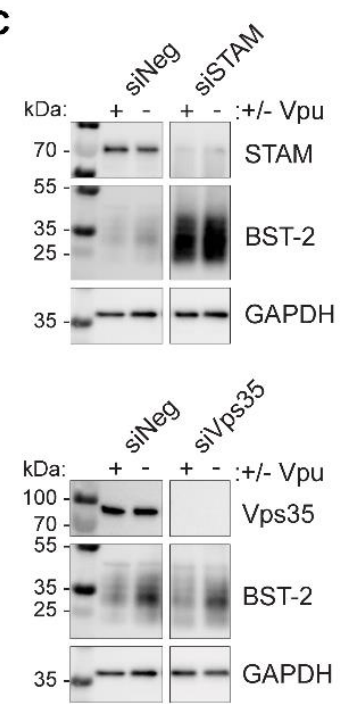

D

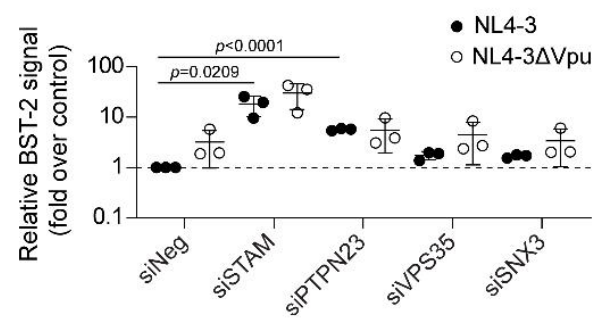

B
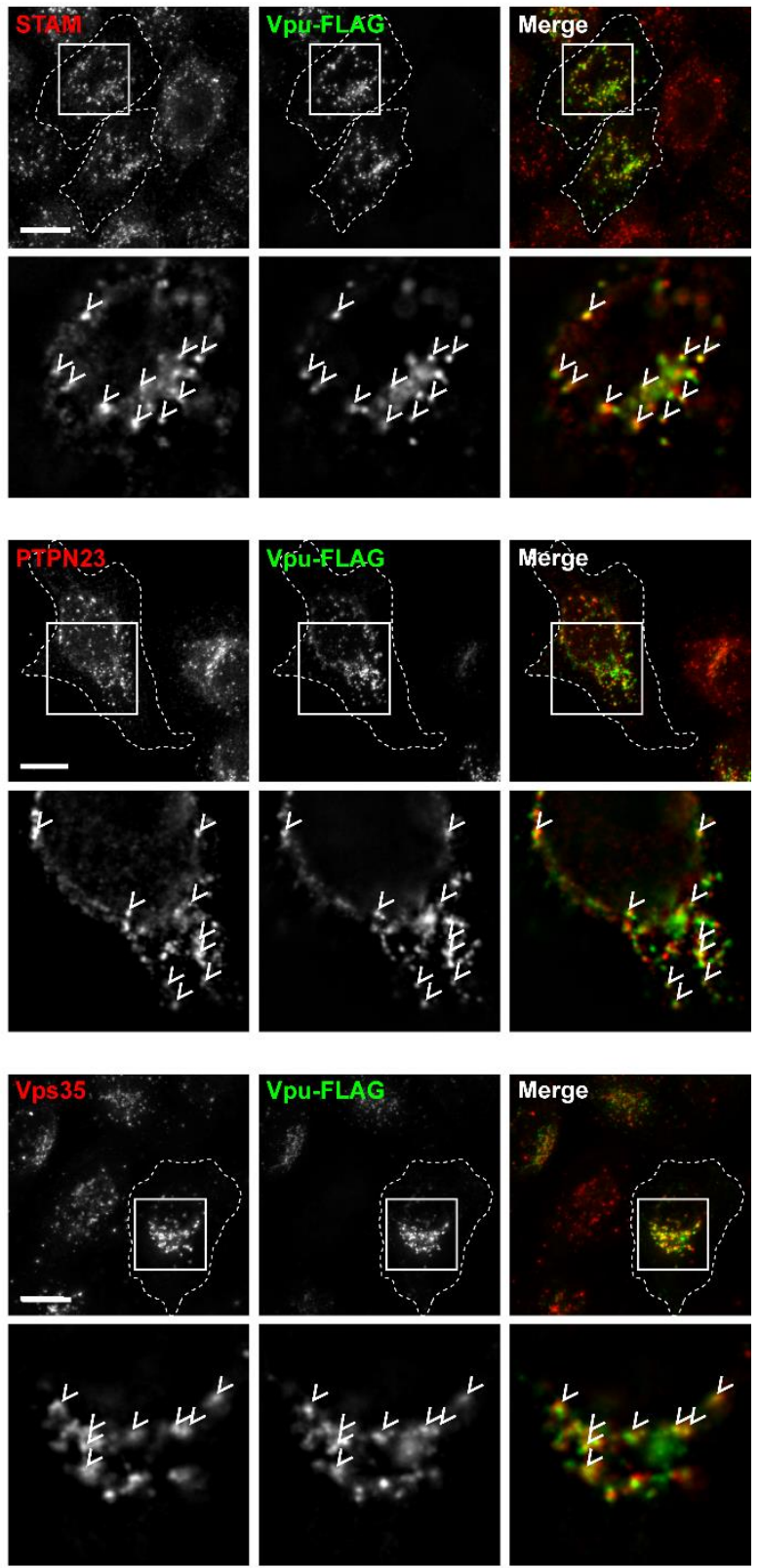

Figure 8. Potential Vpu cofactors: STRING relationships, co-localization with Vpu, and effects on

721 expression of the Vpu-target BST-2. (A) The interactions of proteins in k-means cluster 6 (Figure 6) were

722 visualized using the STRINGdb (Cytoscape) network analysis tool. The interrelated proteins identified

723 represent candidate Vpu cofactors. (B) Immunofluorescence microscopy of Vpu-FLAG and some of the

724 candidate cofactors. HeLa P4.R5 cells were transfected to express Vpu-FLAG. Cells were fixed and

725 stained for the indicated endogenous proteins. Images are z-stack projections of full cell volumes; insets 
726 show single z-sections, with arrows indicating colocalized foci. Scale bars are $10 \mu \mathrm{m}$. (C) Candidate

727 cofactor proteins were transiently knocked-down using siRNAs in HeLa P4.R5 cells. The cells were

728 transfected with pNL4-3 (an HIV proviral plasmid expressing the complete viral genome including Vpu)

729 or pNL4-3 $\mathrm{Vpu} 48$ hours later. The cells were lysed 24 hours post-transfection and BST-2 was probed by

730 SDS-PAGE and western blotting. (D) BST-2 signals were measured relative to loading control (GAPDH)

731 and presented as fold signal over NL4-3 (negative control siRNA; Vpu-expressed). Data are mean +/- SD

732 of three independent experiments; $p$-value determined by Student's $t$-test. 

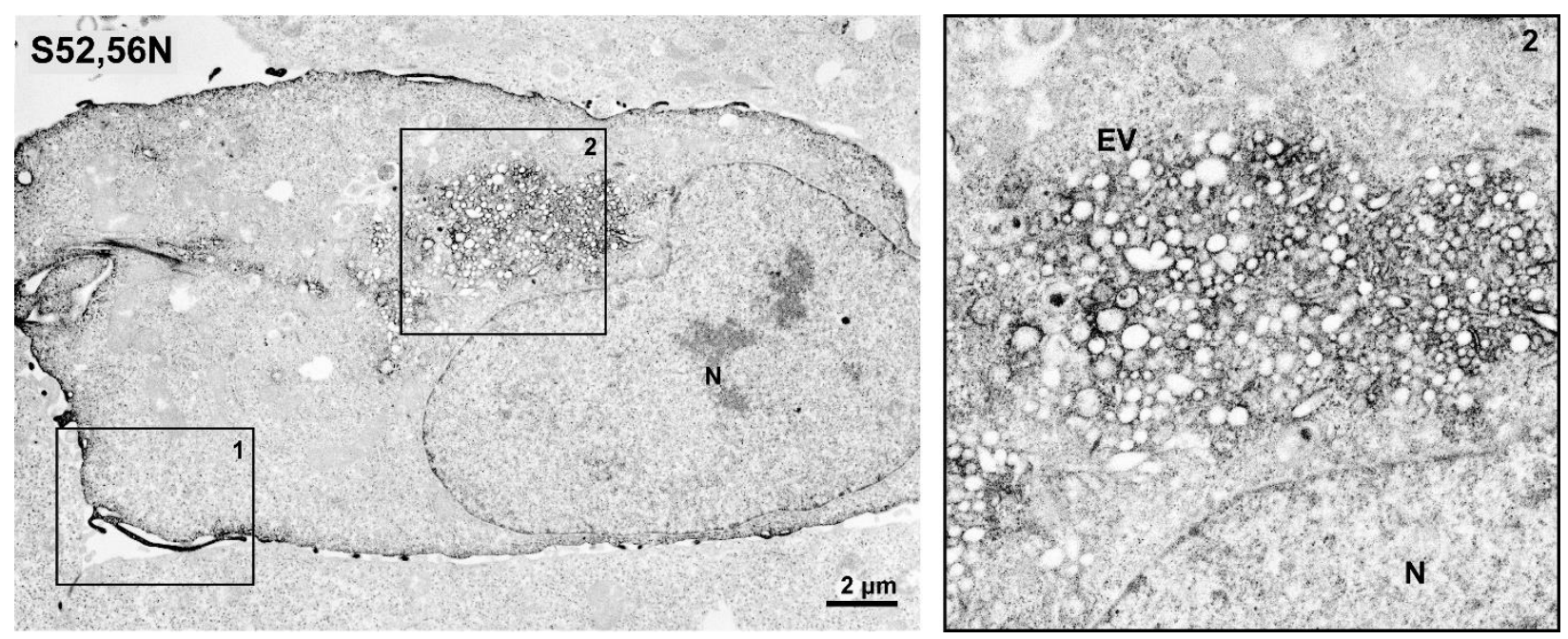

Figure S1. Juxtanuclear endosomal distortion in cells expressing Vpu-S52,56N-APEX2. HeLa P4.R5 cells

were transfected to express Vpu-S52,56N-APEX2. 24 hours later the cells were fixed before APEX2-

737 sections collected and analysed by TEM. The mutant Vpu-S52,56N was localized to the plasma nuclear enlarged vesicles (EV, region 2), similar to the WT Vpu. 

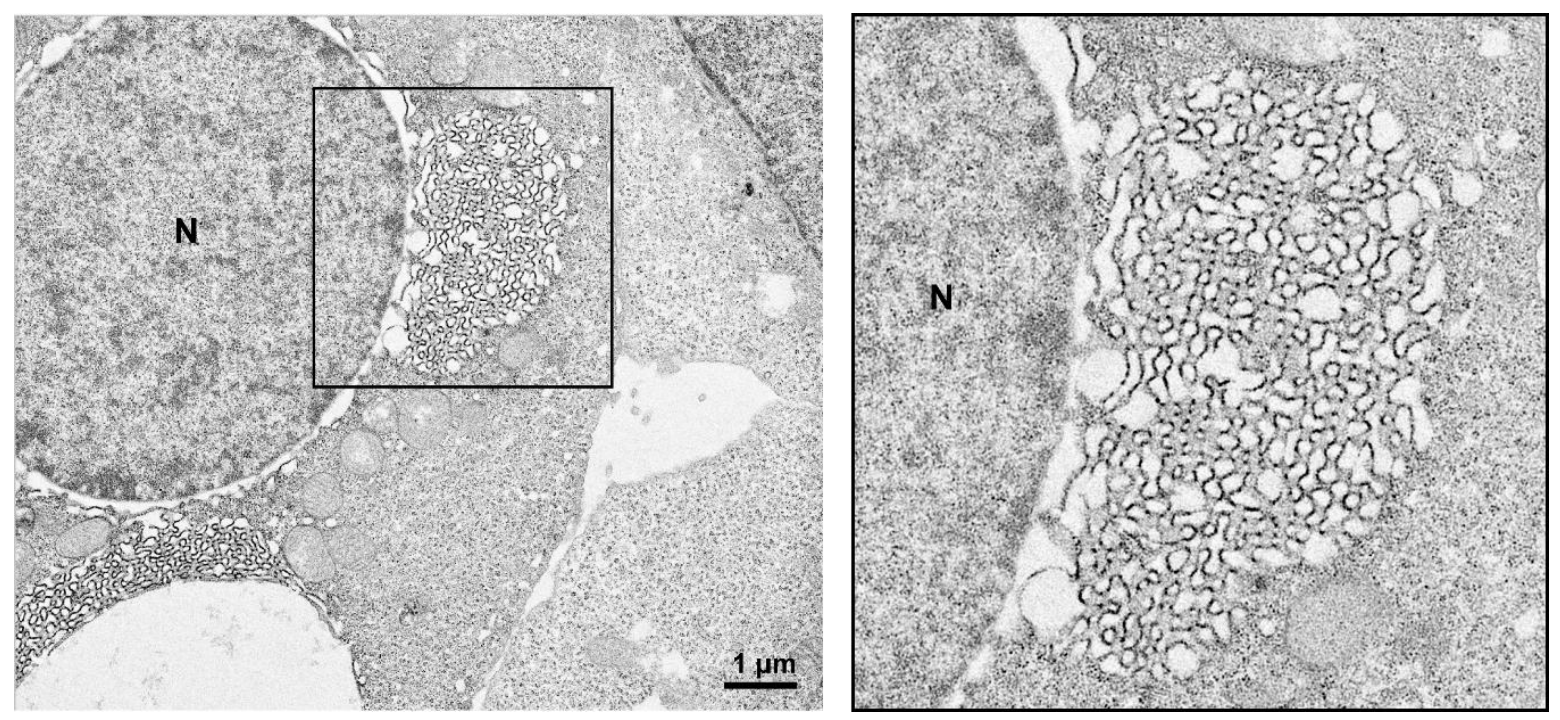

741 Figure S2. Exuberant ER membranes in cells expressing Vpu-A18H-APEX2. HeLa P4.R5 cells were

742 transfected with a VpHu-A18H construct bearing a C-terminal APEX2 tag. 24 hours later the cells were

743 fixed before APEX2-dependent polymerization of DAB and osmium staining. Cells were embedded in

744 resin and $70 \mathrm{~nm}$ sections collected and analysed by TEM. The endoplasmic reticulum-trapped mutant,

$745 \mathrm{~A} 18 \mathrm{H}$, was restricted to the nuclear envelope (NE) and ER and induced membrane reorganisation: when

746 expressed at high levels, the nuclear envelope was distorted by the accumulation of convoluted, smooth

747 membranes. 
A

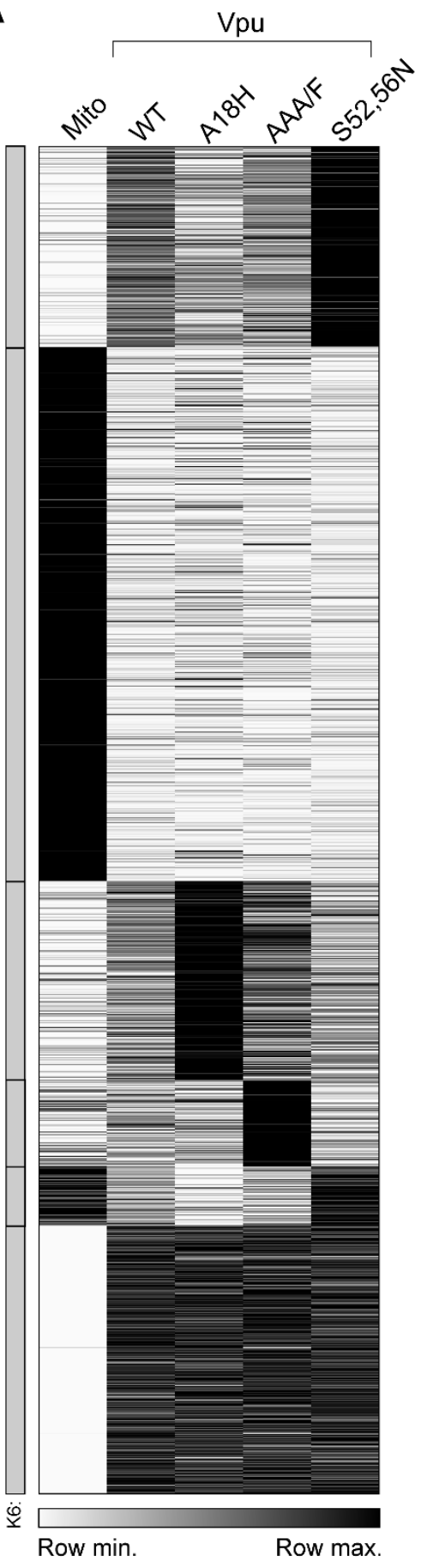

B

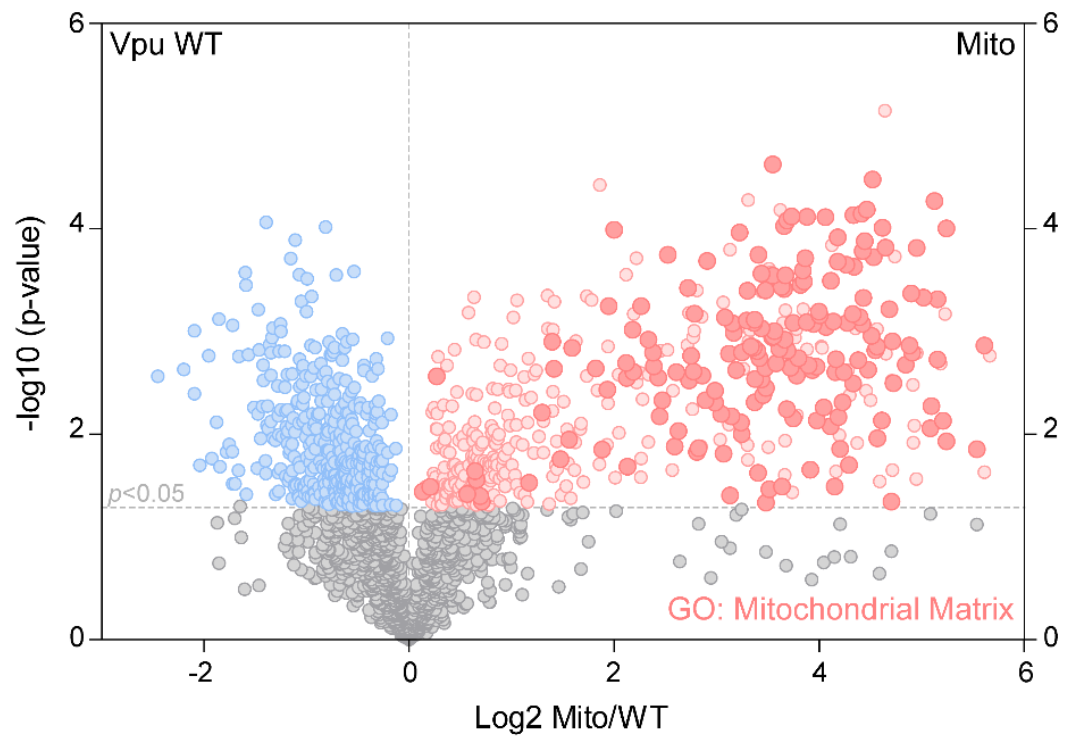

C

Enriched Mito/Vpu WT: $-\log 10$ ( $p$-value)

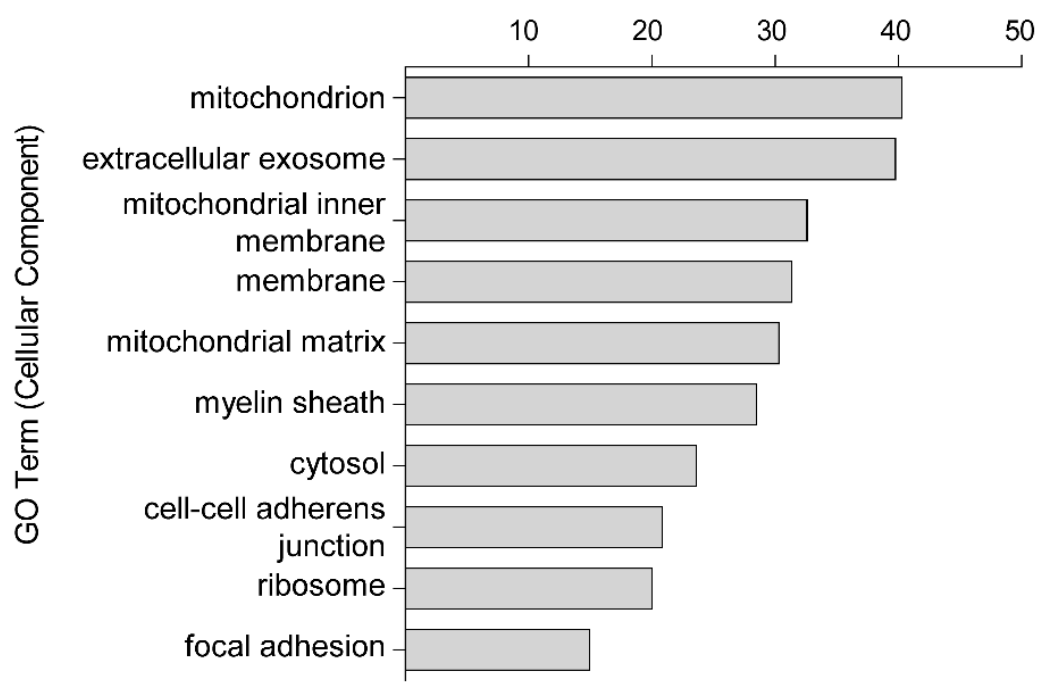

Figure S3. Pair-wise comparison of proximity-ome of Vpu-APEX2 compared to the Mito-APEX2. HeLa

751 in duplicate. Following proximity biotinylation reactions, the biotinylated proteins were isolated and

752 subject to quantitative mass spectrometry. (A) Heatmap showing relative protein abundance across 
753 Mito control and Vpu WT and mutant samples, sorted into 6 k-means clusters (cluster number derived

754 from elbow method). (B) Volcano plot of proteins biotinylated by Vpu-APEX2 vs. Mito-APEX2 control.

755 Mitochondrial proteins corresponding to GO term Mitochondrial Matrix are highlighted. The x-axis

756 shows $\log 2$ fold change and y-axis $-\log 10 p$-value derived from Student's $t$-test. (C) GO enrichment

757 analysis of proteins significantly enriched by Mito-APEX compared to Vpu WT, the top ten GO (cell

758 component) terms are shown. 

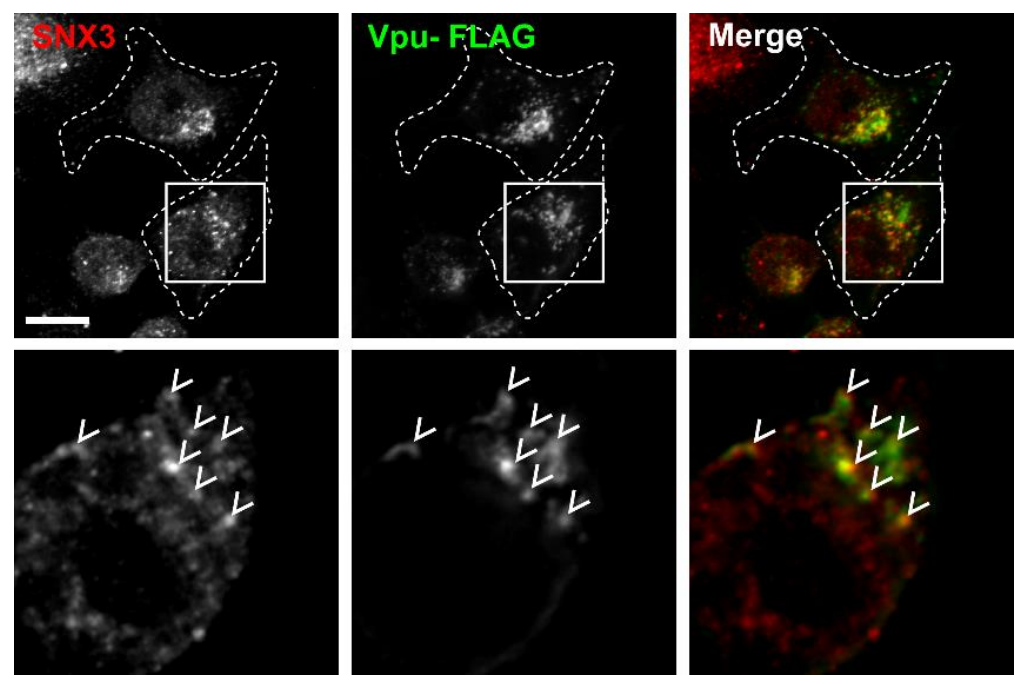

760 Figure S4. Immunofluorescence microscopy of Vpu-FLAG and candidate cofactor SNX3. HeLa P4.R5

761 cells were transfected to express Vpu-FLAG. Cells were fixed and stained for endogenous SNX3 protein

76224 hours post-transfection. Images are z-stack projections of full cell volumes; insets show single z-

763 sections, with arrows indicating colocalized foci. Scale bars are $10 \mu \mathrm{m}$. Some punctate colocalization of

764 Vpu and SNX3 was observed in the perinuclear region, in agreement with immunofluorescent stain of

$765 \quad$ Vpu and retromer component Vps35. 